\title{
Coronary Magnetic Resonance Angiography in Chronic Coronary Syndromes
}

\author{
Reza Hajhosseiny ${ }^{1,2 *}$, Camila Munoz ${ }^{1}$, Gastao Cruz ${ }^{1}$, Ramzi Khamis ${ }^{2}$, Won Yong Kim ${ }^{3}$, \\ Claudia Prieto ${ }^{1,4}$ and René M. Botnar ${ }^{1,4,5}$
}

${ }^{1}$ School of Biomedical Engineering and Imaging Sciences, King's College London, London, United Kingdom, ${ }^{2}$ National Heart and Lung Institute, Imperial College London, London, United Kingdom, ${ }^{3}$ Department of Cardiology and Institute of Clinical Medicine, Aarhus University Hospital, Skejby, Denmark, ${ }^{4}$ Escuela de Ingeniería, Pontificia Universidad Catolica de Chile,

Santiago, Chile, ${ }^{5}$ Instituto de Ingeniería Biologica y Medica, Pontificia Universidad Catolica de Chile, Santiago, Chile

\section{OPEN ACCESS}

Edited by:

Bernhard L. Gerber

Cliniques Universitaires

Saint-Luc, Belgium

Reviewed by:

David Winkel,

University Hospital of

Basel, Switzerland

Thoralf Niendorf

Helmholtz Association of German

Research Centers (HZ), Germany

*Correspondence:

Reza Haihosseiny

reza.hajhosseiny@kcl.ac.uk orcid.org/0000-0002-8116-460X

Specialty section:

This article was submitted to

Cardiovascular Imaging,

a section of the journal

Frontiers in Cardiovascular Medicine

Received: 19 March 2021

Accepted: 23 July 2021

Published: 17 August 2021

Citation:

Hajhosseiny R, Munoz C, Cruz G,

Khamis $R$, Kim WY, Prieto $C$ and

Botnar RM (2021) Coronary Magnetic

Resonance Angiography in Chronic

Coronary Syndromes.

Front. Cardiovasc. Med. 8:682924.

doi: 10.3389/fcvm.2021.682924
Cardiovascular disease is the leading cause of mortality worldwide, with atherosclerotic coronary artery disease (CAD) accounting for the majority of cases. X-ray coronary angiography and computed tomography coronary angiography (CCTA) are the imaging modalities of choice for the assessment of CAD. However, the use of ionising radiation and iodinated contrast agents remain drawbacks. There is therefore a clinical need for an alternative modality for the early identification and longitudinal monitoring of CAD without these associated drawbacks. Coronary magnetic resonance angiography (CMRA) could be a potential alternative for the detection and monitoring of coronary arterial stenosis, without exposing patients to ionising radiation or iodinated contrast agents. Further advantages include its versatility, excellent soft tissue characterisation and suitability for repeat imaging. Despite the early promise of CMRA, widespread clinical utilisation remains limited due to long and unpredictable scan times, onerous scan planning, lower spatial resolution, as well as motion related image quality degradation. The past decade has brought about a resurgence in CMRA technology, with significant leaps in image acceleration, respiratory and cardiac motion estimation and advanced motion corrected or motion-resolved image reconstruction. With the advent of artificial intelligence, great advances are also seen in deep learning-based motion estimation, undersampled and super-resolution reconstruction promising further improvements of CMRA. This has enabled high spatial resolution (1 $\mathrm{mm}$ isotropic), 3D whole heart CMRA in a clinically feasible and reliable acquisition time of under $10 \mathrm{~min}$. Furthermore, latest super-resolution image reconstruction approaches which are currently under evaluation promise acquisitions as short as $1 \mathrm{~min}$. In this review, we will explore the recent technological advances that are designed to bring CMRA closer to clinical reality.

Keywords: coronary angiography, CMRA, CCS, atherosclerosis, plaque, magnetic resonance imaging

\section{INTRODUCTION}

Coronary artery disease (CAD) is the leading cause of cardiovascular morbidity and mortality worldwide $(1,2)$. CAD is predominantly caused by coronary atherosclerosis, which initiates in the early decades of life and progresses at a variable pace depending on the intrinsic propensity of the individual in combination with lifestyle modifications and therapeutic interventions (3-5). 
The clinical manifestations of CAD can present either as an acute process or a more stable but chronic deterioration of symptoms. The European Society of Cardiology has recently categorised CAD as either acute coronary syndromes (ACS) or chronic coronary syndromes (CCS) (6). Whilst cardiovascular magnetic resonance $(\mathrm{CMR})$ has a clear role in the diagnosis and management of patients with ACS, this is beyond the scope of this review. We will instead focus on the role of coronary magnetic resonance angiography (CMRA) in patients with CCS with a particular emphasis on the current status quo and future directions.

Chronic coronary syndromes can present clinically in a variety of ways; symptoms of stable angina (e.g., chest pain and/or dyspnoea), symptoms of heart failure or asymptomatic left ventricular impairment, symptomatic and asymptomatic cardiac arrhythmias (6). The early detection of CCS and targeted risk stratification will facilitate the timely initiation of therapeutic interventions and monitoring of disease progression.

$\mathrm{X}$-ray coronary angiography is the reference standard imaging modality for the assessment of CAD with unrivalled temporal and spatial resolution, as well as the versatility to enable real time coronary intervention for patients presenting with ACS. The addition of functional physiological assessment of CAD with pressure wiring is particularly helpful in patients presenting with CCS and has been shown to be prognostically significant
$(7,8)$. Plaque characterisation and coronary vascular anatomy can be further complemented with intravascular imaging (9, 10). However, risk from ionising radiation, viscous iodinecontaining radiocontrast agent induced acute kidney injury and its potential progression to chronic kidney disease (11) as well as potential invasive complications limit the surveillance suitability of invasive assessment for CCS beyond the initial diagnosis.

Computed tomography coronary angiography (CCTA) has emerged as a credible non-invasive alternative for the assessment of patients with CCS. It offers high diagnostic accuracy in terms of comprehensive assessment of luminal stenosis, fractional flow reserve and plaque characterisation (12-15). However, it is also limited by ionising radiation, iodinated nephrotoxic contrast agents and calcium related blooming artefacts, dampening its suitability in patients with highly calcified plaque lesions or for the longitudinal assessment of CAD.

Coronary magnetic resonance angiography could potentially offer a non-invasive alternative for the early detection and longterm monitoring of CCS, which is free of ionising radiation and iodinated nephrotoxic contrast agents. It could also be combined with volumetric assessment of left ventricular function, myocardial perfusion, myocardial tissue characterisation, valvular assessment and coronary plaque characterisation to brand CMR as one of the most comprehensive imaging modalities for cardiovascular assessment. Here we discuss the
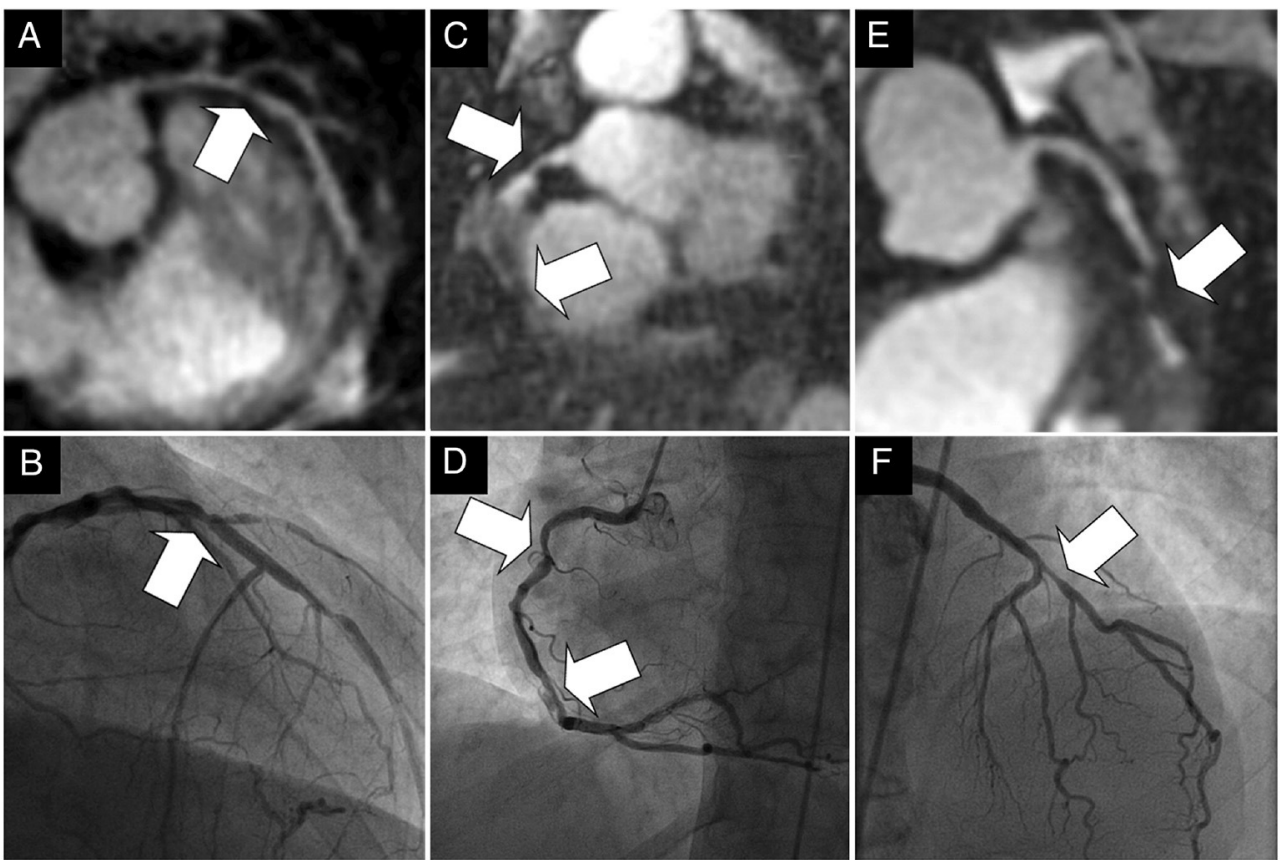

FIGURE 1 | (A-F) Examples of the comparison between multiplanar reformats of the whole-heart 1D self-navigated CMRA data sets (top row) and the corresponding $\mathrm{x}$-ray coronary angiograms (bottom row) in three patients. The lesion in the proximal LAD artery and just distal to the take-off of a diagonal branch can clearly be identified in the first patient in (A). while this is confirmed on the x-ray angiogram in (B). While the luminal narrowing of the proximal RCA in the second patient on (C). can clearly be identified, the further course of this artery is obscured in the region of a stent. The same in stent restenoses can be identified in (D). In the third patient in (E). significant disease is identified in the proximal LAD artery at CMRA and is confirmed on, (F). the corresponding $\mathrm{X}$-ray coronary angiogram. Arrows = stenoses; $L A D$, left anterior descending artery; RCA, right coronary artery. Adapted with permission from Piccini et al. (46). 

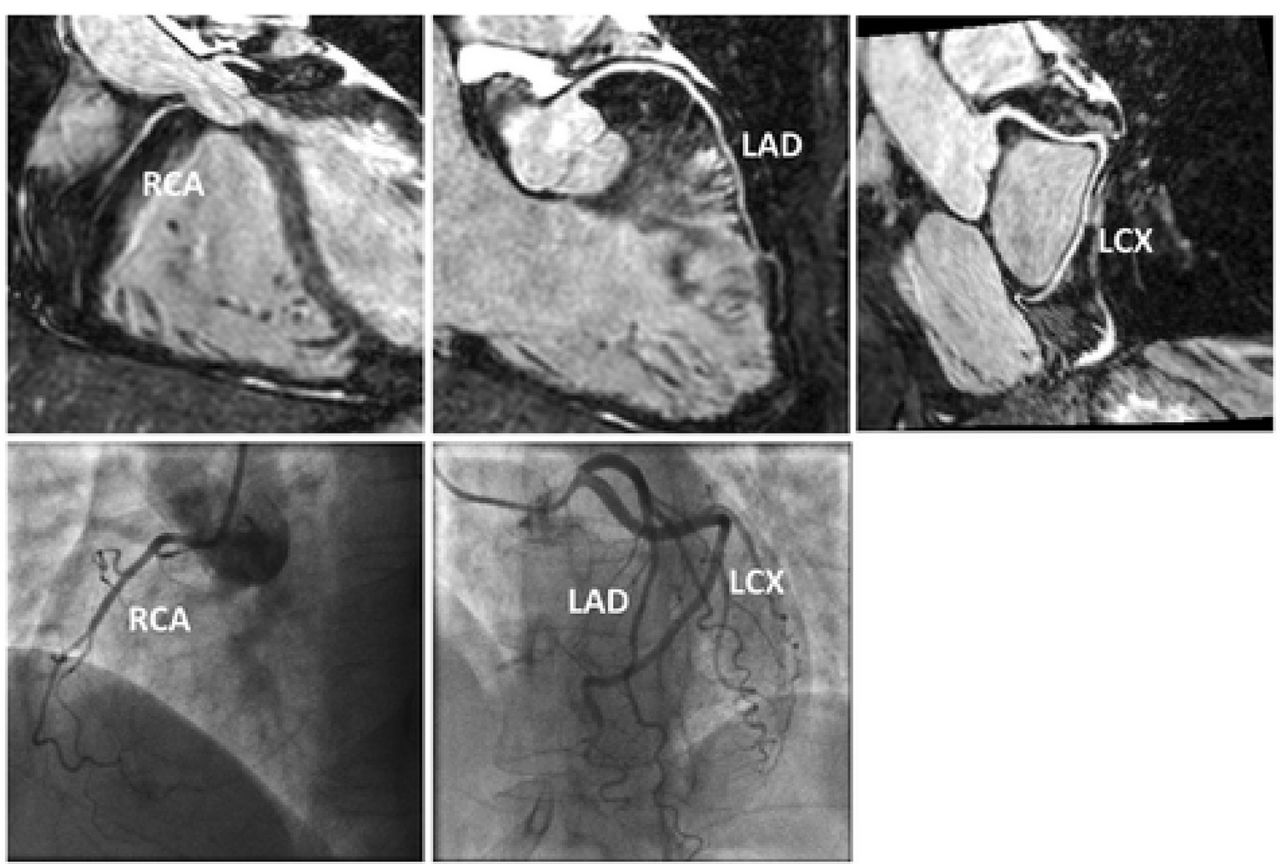

FIGURE 2 | Reformatted CMRA datasets (top row) from a patient without coronary artery disease but non dominant RCA. Coronary x-ray angiography in the same patient (bottom row). RCA, right coronary artery; LAD, left anterior descending artery; LCX, left circumflex artery. Adapted with permission from Henningsson et al. (52).

current status quo and potential future role of CMRA as a viable alternative for the assessment of patients with CCS.

\section{CORONARY MAGNETIC RESONANCE ANGIOGRAPHY}

The potential of CMRA to exclude significant CAD was first demonstrated two decades ago in a multicentre study of 109 patients, which compared non-contrast CMRA against invasive $\mathrm{X}$-ray angiography (16). In this study, the sensitivity, specificity, positive and negative predictive values, and accuracy of CMRA were $93,42,70,81$, and $72 \%$ respectively. These findings were confirmed in a subsequent study of 127 patients comparing CMRA vs. invasive X-ray angiography (17). In this study, the sensitivity, specificity, positive and negative predictive values, and accuracy of CMRA were $88,72,71,88$, and $79 \%$ respectively. In a direct head-to-head comparison of CMRA vs. CCTA in patients with suspected CAD who were also assessed with invasive X-ray coronary angiography, there was no significant difference between CCTA and CMRA in terms of diagnostic accuracy, suggesting that CMRA could be used in these patients to exclude significant disease, inform revascularization and if the CMRA scan was negative the event free survival rate was comparable with CCTA $(18,19)$. To assess the prognostic value of CMRA, 207 patients with suspected CAD who underwent noncontrast whole-heart CMRA were followed up by Yoon et al. (20). Coronary stenosis was significantly associated with major adverse cardiac events (myocardial infarction, cardiac death, and unstable angina) and all cardiac events (which included revascularization $>90$ days after CMRA).

However, despite the early promise of CMRA, clinical application is currently confined to a few specialised centres within a set of niche indications e.g., suspected anomalous coronary arteries, coronary artery aneurysms and assessment of the proximal coronary arteries. Reasons for the slow uptake of CMRA in clinical practise include lower spatial resolution compared to CCTA and invasive X-ray angiography, significantly longer and unpredictable acquisition time, complicated scan planning, and motion related (cardiac and respiratory) image quality degradation.

Significant technological strides in CMR image acquisition and image processing are now enabling some of these technical challenges to be overcome. The most notable breakthroughs are in the fields of respiratory motion compensation and image acceleration. Further recent advances in deep learning-based motion correction and image reconstruction could potentially enable 3D whole-heart CMRA with similar spatial resolution and acquisition time as CCTA in the future. We will discuss some of these recent developments in the following sections.

\section{CARDIAC AND RESPIRATORY MOTION COMPENSATION}

In order to acquire a high spatial resolution $3 \mathrm{D}$ whole-heart CMRA, patients are required to be continuously scanned for several minutes with data acquired from numerous heat beats under free breathing. This scenario produces a challenging 


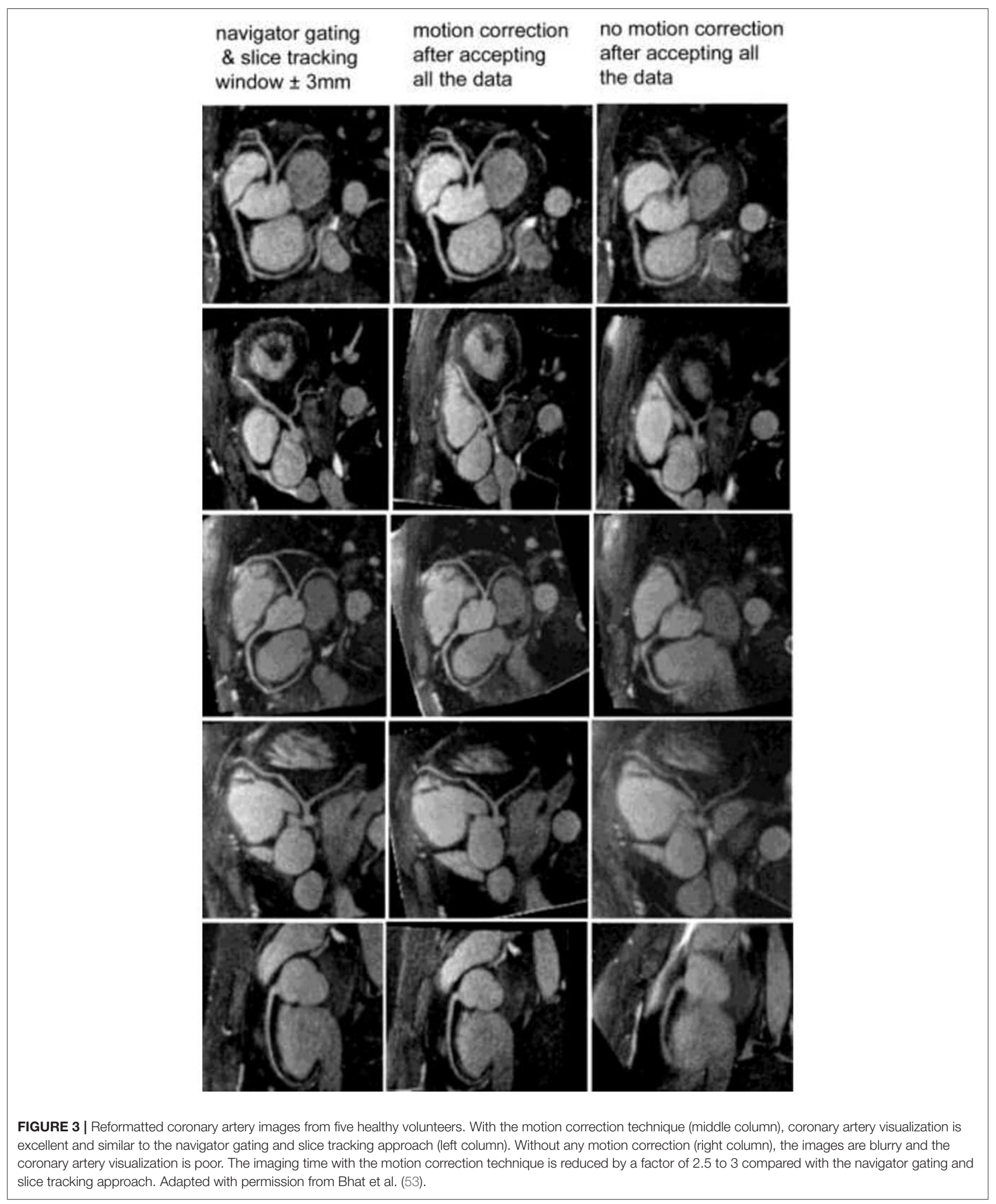




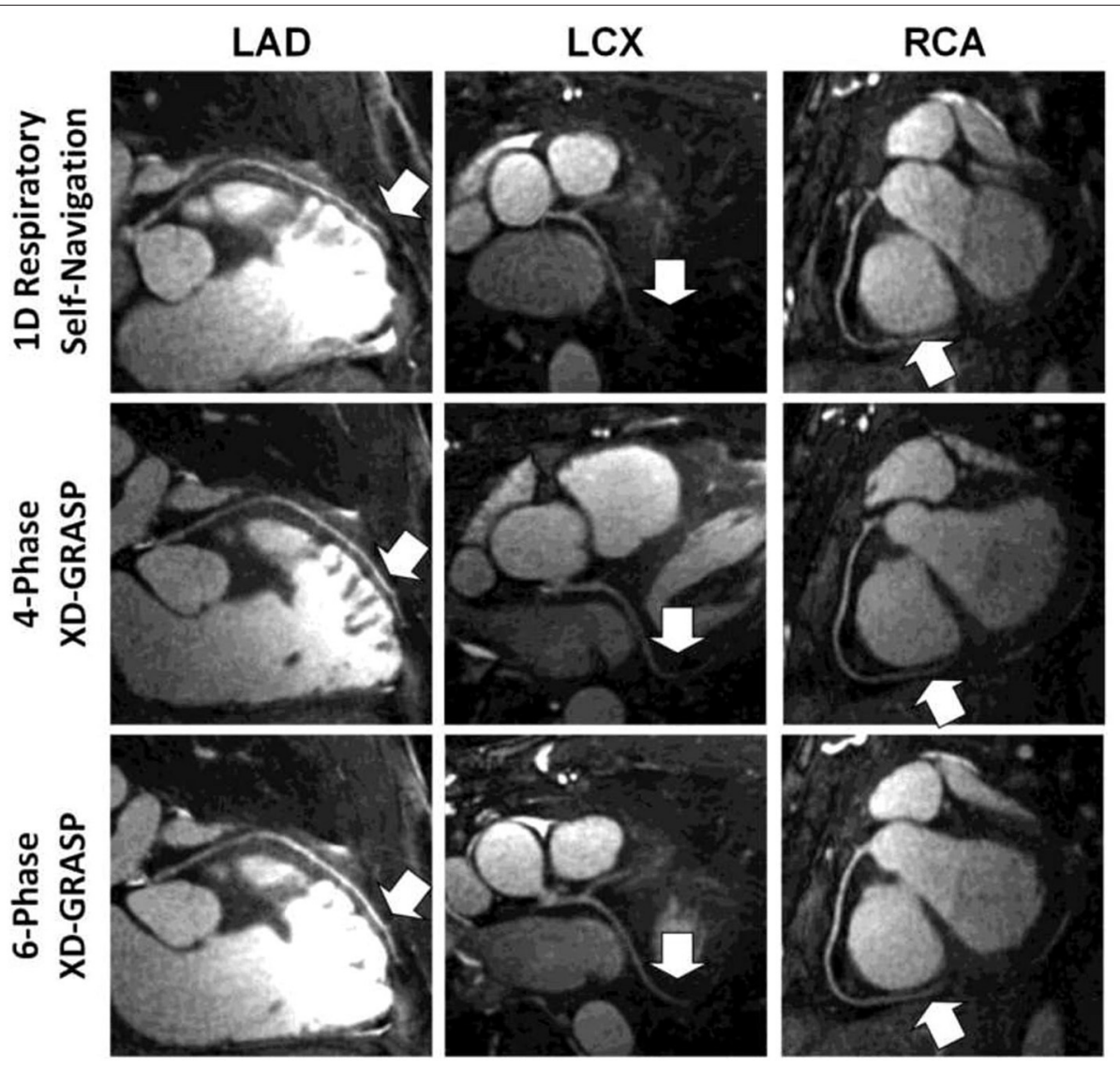

FIGURE 4 | Examples of multiplanar reformatted coronary arteries from one representative healthy adult volunteer. Although respiratory self-navigated reconstruction with 1D motion correction could achieve high image quality (top row), a clear improvement in sharpness as well as visible vessel length (arrows) can be noticed in both four-phase (middle row) and six-phase (bottom row) extradimensional golden-angle radial sparse parallel (XD-GRASP) reconstructions. Adapted with permission from Piccini et al. (54).

multi-dimensional cardiac and respiratory motion environment, which needs to be compensated through image acquisition and reconstruction techniques. Cardiac motion artefacts are typically minimised by prospective electrocardiographic (ECG) triggering to acquire data during mid-to-late diastole. However, this may be susceptible to image degradation as a result of cardiac arrhythmias, heart rate variability or user dependent estimation of the resting period. Systolic image acquisition is less sensitive to arrhythmias and heart rate variability. However, longer acquisition times may be needed due to a shorter resting period compared to diastolic imaging. Alternatively, data can be continuously acquired and then retrospectively reconstructed into multiple cardiac phases, thus selecting the phase with the minimum cardiac motion artefact, similar to CCTA $(21,22)$.

Various techniques have been proposed to compensate for respiratory motion artefacts during CMRA acquisition. The most rudimentary techniques utilise breath-holding to minimise respiratory motion artefacts (23-26), which enable both $2 \mathrm{D}$ and 3D CMRA acquisitions within a single albeit long breath-hold (27-29). However, image quality is frequently suboptimal due to diaphragmatic drift and difficulty of patients to hold their breath for a prolonged period $(30,31)$. As a result, clinically established 3D CMRA techniques implement free-breathing motion compensated protocols $(30,32)$. Initially, respiratory bellows (33) and amplitude demodulation of the ECG signal (34), which are external respiratory monitoring devices were used to enable free breathing CMRA. This is called respiratory gating as data is only acquired at end expiration when respiratory motion is minimal. However, this method is both labour intensive and time consuming to implement. Furthermore, it is not possible to estimate the true multidimensional extend of respiratory motion. As a result, respiratory navigators, incorporating the intrinsic CMR signal to track respiratory related movement of the heart became the dominant form of respiratory gating/compensation for free breathing CMRA. Here, the diaphragmatic 1D navigator $(35,36)$ takes advantage of the liver-diaphragm interface to perform respiratory translational motion estimation, which enables both respiratory gating and 1D (superior-inferior) motion correction. However, there is a non-linear relationship (37) between the movement of the liver/diaphragm and the heart, which is patient specific and subject to hysteresis (the motion of breathing-in is different than breathing-out). Despite 
A
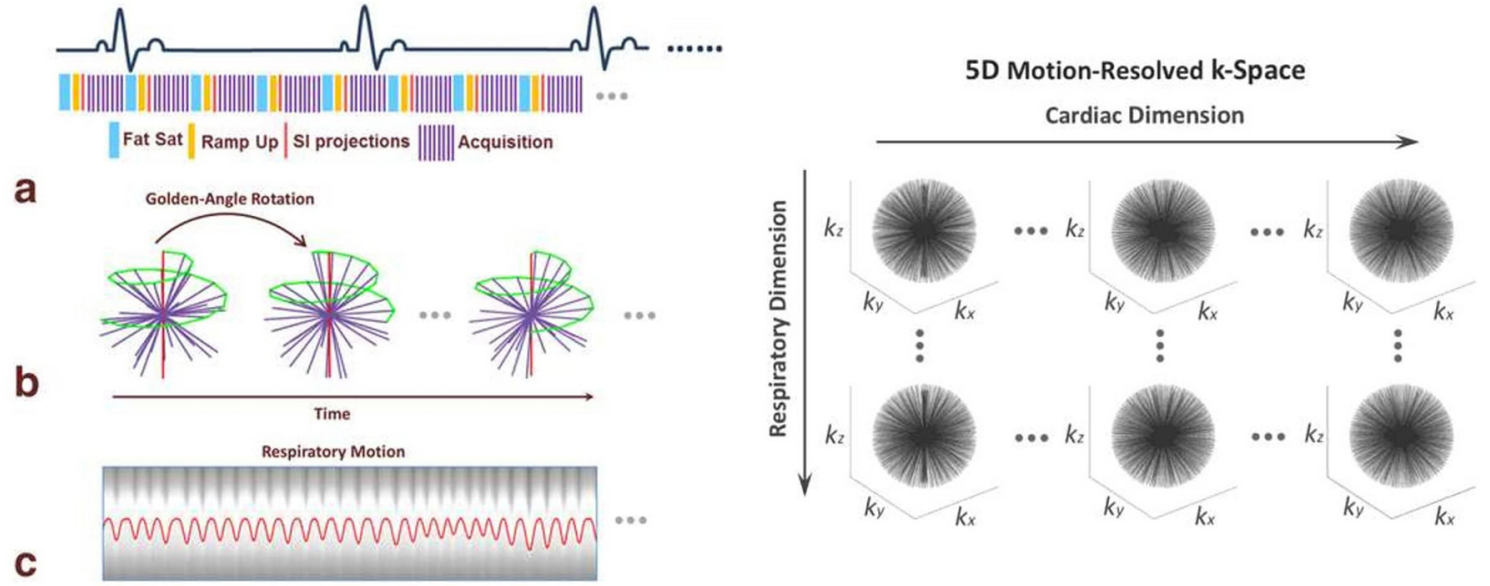

FIGURE 5 | (A) Data acquisition scheme and respiratory motion extraction for non-ECG-triggered whole-heart imaging. (a) A 3D radial b-SSFP sequence that is segmented into multiple interleaves (purple lines) is used for MR data acquisition. Each interleave starts with a spoke oriented along the superior to inferior direction for self-navigation (red lines) and is preceded by fat saturation (blue lines). Ten additional ramp-up RF pulses (yellow lines) are deployed between the fat saturation module and the data acquisition window to restore restoring steady-state at each interleave. (b) 3D radial sampling trajectory based on the spiral phyllotaxis pattern. Each interleave is rotated by the golden-angle $\left(137.51^{\circ}\right.$ ) about the $z$-axis, starting with a self-navigation spoke (red lines) for respiratory motion extraction. (c) An extracted respiratory motion signal is superimposed to the 1D FFT of an example series of SI readouts. (B) The acquired k-space is sorted into a 5D dataset (kx-ky $-k_{z}-c a r d i a c$ phase-respiratory phase) using respiratory motion signal extracted from self-navigators and cardiac motion signal obtained from recorded ECG time stamp. The datasets are first binned into different cardiac phases with a desired temporal resolution, then each cardiac phase is further sorted into multiple respiratory motion phases spanning from end-expiration to end-inspiration. The data sorting process is performed such that the number of spokes grouped in each temporal phase is the same. SSFP, Steady State Free Precession; MR, Magnetic Resonance; RF, Radiofrequency; FFT, fast Fourier transform; SI, Superior-Inferior; ECG,

Electrocardiogram. Adapted with permission from Feng et al. (55).

this, a population derived linear correction factor of 0.6 is used as an approximation of the motion between liver/diaphragm and the heart $(38,39)$. Furthermore, precise planning of the diaphragmatic navigator is complex and requires expertise, acquisition times are often unpredictable and reliant on the breathing pattern of patients and it is highly inefficient $(\sim 30-$ $50 \%)$ as data is acquired only at end expiration within a very narrow acquisition window of $\pm 5 \mathrm{~mm}(30,36)$.

Several novel respiratory motion compensation frameworks have recently been proposed to overcome some of these drawbacks, principally to deal with the 3D nature of respiratory motion, improve image quality, increase respiratory scan efficiency to $100 \%$ and therefore significantly reduce acquisition times. Using the so called "1D self-navigation" approach (40), the acquired CMR data is used to estimate displacement/movement of the heart induced by respiratory motion $(40,41)$, eliminating the need for the correction factor, enabling translational motion correction and acquiring data at every point of the respiratory cycle which enables $100 \%$ respiratory scan (42-45). In a cohort of 78 patients, a self-navigated CMRA framework enabled 92, 84 , and $56 \%$ of proximal, middle and distal coronary segments, respectively, to be visualised, with a per vessel sensitivity and specificity for stenosis ( $>50 \%$ ) detection of 65 and $85 \%$ compared with X-ray coronary angiography (Figure 1) (46).

However, with 1D self-navigation, it is difficult to separate moving (e.g., heart) from static (e.g., chest wall) tissue, which introduces artefacts (47). Image-based navigators (iNAVs) are an alternative platform to respiratory self-navigation. Whilst offering up to $100 \%$ respiratory scan efficiency, the principle aim of iNAVs is to separate moving tissue from static tissue by acquiring a low spatial resolution $2 \mathrm{D} / 3 \mathrm{D}$ image at every heartbeat prior to the acquisition of high resolution CMRA (47-51). This framework also enables the capability to estimate respiratory motion in multiple directions to factor in the multidimensional motion of the heart. Furthermore, it eliminates additional planning as the iNAV can be derived within the same field of view (FOV) and orientation as the CMRA planning. An early version of iNAV CMRA framework demonstrated highly diagnostic image quality in the proximal, middle and distal coronary segments (98, 94, and 91, respectively) (Figure 2) (52). The sensitivity, specificity, and negative predictive values were 86 , 83 , and $95 \%$ per patient, 80,92 , and $97 \%$ per vessel and 73,95 , and $98 \%$ per segment; compared with X-ray coronary angiography.

\section{CORONARY MAGNETIC RESONANCE ANGIOGRAPHY TECHNIQUES CURRENTLY IN DEVELOPMENT}

Despite offering $100 \%$ respiratory scan efficiency, self-navigation or iNAV frameworks on their own are not sufficient. It can take up to $30 \mathrm{~min}$ to acquire a fully sampled high resolution $(\approx 1 \mathrm{~mm}$ 

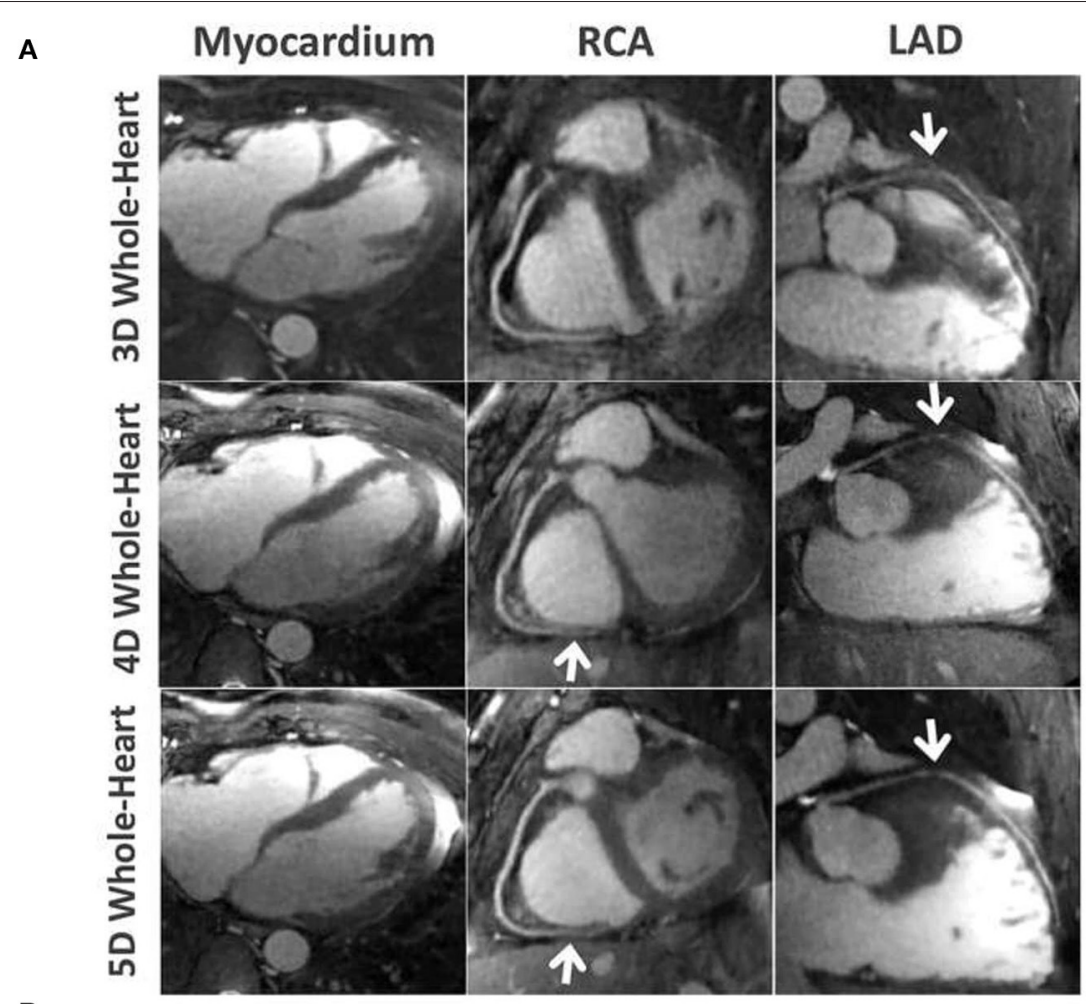

B

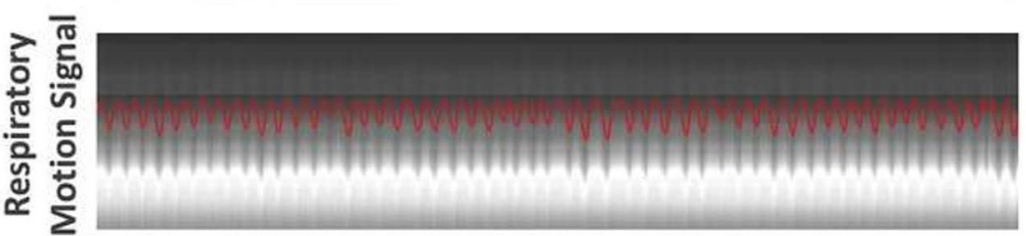

FIGURE 6 | (A) Comparison of the myocardium, the RCA and LAD coronary arteries for different imaging techniques in one subject. 5D whole-heart images (end-expiratory phase) achieved improved visual delineation of the myocardial wall and different segments of the coronary arteries (white arrows) over 4D whole-heart images, and improved delineation of the LAD over self-navigated 3D whole-heart images. (B) Corresponding respiratory motion pattern extracted from the continuous acquired whole-heart dataset in this subject. RCA, right coronary artery; LAD, left anterior descending artery. Adapted with permission from Feng et al. (55).

isotropic) CMRA. Here we will discuss the novel techniques in development which incorporate self-navigation or iNAV systems with a combination of image acceleration techniques (undersampled acquisition, parallel imaging, iterative non-linear reconstruction) to achieve high-spatial resolution CMRA within a clinically feasible acquisition time. We will also touch on more advanced motion correction frameworks which factor in the more complex 3D non-rigid motion of the heart within the thoracic cavity. Finally, we will briefly discuss emerging deep learning super resolution CMRA reconstruction frameworks that could potentially enable 3D whole-heart CMRA with similar spatial resolution and acquisition time as CCTA.

It is possible to combine an undersampled $3 \mathrm{D}$ radial trajectory acquisition with $1 \mathrm{D}$ navigators (diaphragmatic or selfnavigation) to allocate the acquired data into specific phases in the respiratory cycle (respiratory bins). Registration algorithms subsequently assess the motion between each bin and a reference end-expiratory bin. Images from all respiratory bins are then motion corrected to the common respiratory position and averaged to produce a respiratory motion corrected image. This methodology allows for $100 \%$ respiratory scan efficiency with a drastically shorter acquisition time in comparison to respiratory gating alone with similar image quality (Figure 3) (22, 40, 41, 53). Respiratory resolved frameworks have been proposed to further reduce the multidirectional non-rigid motion related artefacts (Figure 4) (54), displaying substantial enhancement in vessel length depiction as well as vessel sharpness in comparison to $1 \mathrm{D}$ self-navigation frameworks. This approach has been extended to cardiac phases (so called "5D whole-heart") from free-running frameworks (Figures 5, 6) (21, 55-57). However, lower signal to noise ratio and prolonged reconstruction time (with compressed sensing) are associated with radial sampling. Spiral trajectory k-space CMRA acquisitions have been proposed to improve scan efficiency, the signal to noise ratio and reduce undersampling artefacts by oversampling near the $\mathrm{k}$-space origin (58-60). 


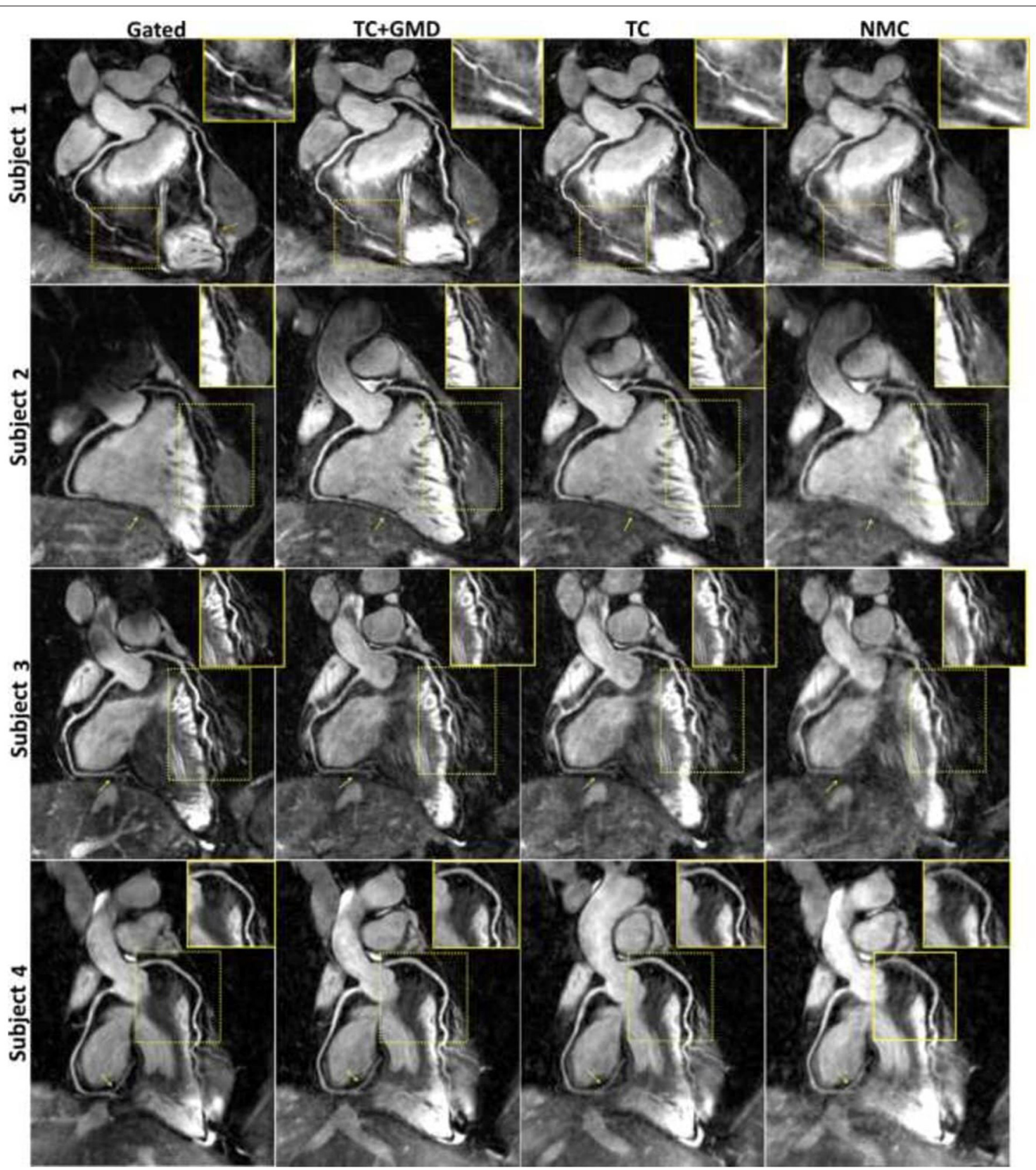

FIGURE 7 | Reformatted coronary lumen images for gated, TC+GMD, TC, and NMC for subjects 1-4. Blurring present in the NMC images is reduced with TC, and sharpness is further increased with TC+GMD (magnified boxes). The distal part of both coronaries is particularly affected by motion (arrows). Note that TC and TC+GMD have image quality similar to that for gated. GMD, general matrix description; TC, translational correction; TC+GMD, two-step translational and non-rigid correction; NMC, non-motion-corrected. Adapted with permission from Cruz et al. (62)

An alternative approach is a golden-step Cartesian trajectory with spiral profile ordering $\mathrm{k}$-space acquisition, which can be combined with iNAVs to enable respiratory binning as described above (61). Using the binned data, it is possible to estimate and subsequently reconstruct the $3 \mathrm{D}$ non-rigid respiratory motion by combining beat-to-beat $2 \mathrm{D}$ translational and bin-to-bin $3 \mathrm{D}$ non-rigid motion correction to a common reference position (Figure 7) (62). This approach can increase the signal to noise ratio compared with radial sampling, whilst also improving image quality compared with $2 \mathrm{D}$ rigid translational motion correction frameworks. To shorten acquisition times and therefore enable higher spatial resolutions, Bustin et al. (63) adapted a highly undersampled patch-based CMR reconstruction framework (64) to propose a 3D patch-based low-rank (PROST) reconstruction, enabling $<1 \mathrm{~mm}^{3}$ spatialresolution free-breathing whole-heart 3D CMRA with $<10$ min 


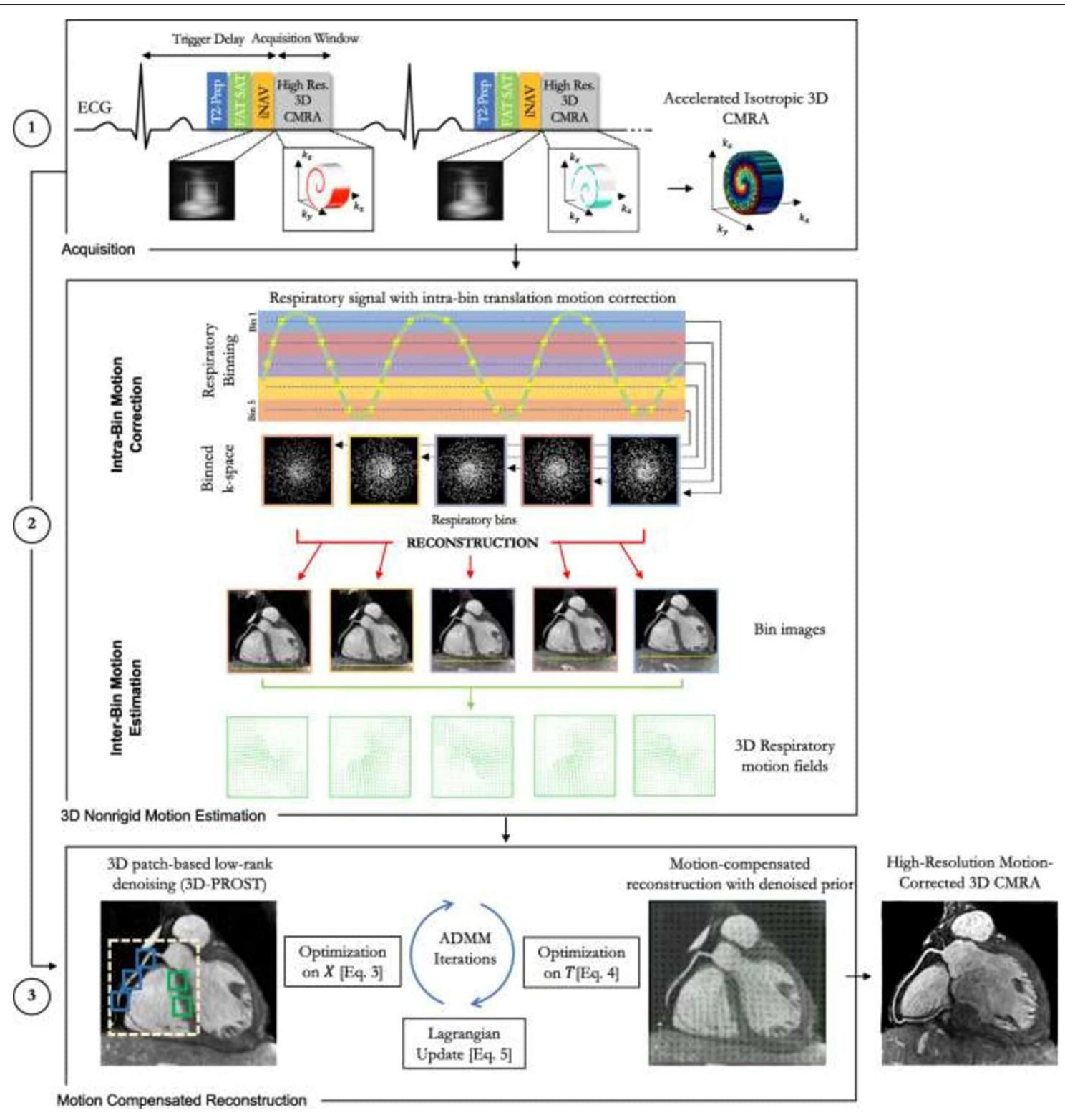

FIGURE 8 | Schematic overview of the accelerated free-breathing 3D CMRA acquisition with sub-millimeter isotropic resolution, 100\% scan efficiency and non-rigid motion-compensated PROST reconstruction. (1) CMRA acquisition is performed with an undersampled 3D variable density spiral-like Cartesian trajectory with golden angle between spiral-like interleaves (VD-CASPR), preceded by 2D image navigators (iNAV) to allow for $100 \%$ scan efficiency and beat-to-beat translational respiratory-induced motion correction of the heart. (2) Foot-head respiratory signal is estimated from the 2D iNAVs and used to assign the acquired data into five respiratory bins and translation-corrected respiratory bins. Subsequent reconstruction of each bin is performed using soft-gated SENSE and 3D non-rigid motion fields are then estimated from the five reconstructed datasets. (3) The final 3D whole-heart motion-corrected CMRA image is obtained using the proposed 3D PROST non-rigid motion-compensated reconstruction. CMRA, coronary magnetic resonance angiography; PROST, patch-based undersampled reconstruction; ADMM, alternating direction method of multipliers. Adapted with permission from Bustin et al. (65).

predictable acquisition time with $100 \%$ scan efficiency. In a validation cohort of healthy subjects, image quality was comparable to the fully sampled acquisition and significantly improved compared to both iterative SENSE and compressed sensing reconstruction methods. This framework has been extended to include bin-to-bin non-rigid respiratory motion correction and has been compared against CCTA in patients with suspected CAD (Figures 8, 9) (65). In a single centre study of 50 patients, this CMRA technique obtained diagnostic image quality in $95,97,97$, and $90 \%$ of all, proximal, middle and distal coronary segments, respectively. Furthermore, 100, 97, 96, and $87 \%$ of left main stem, right coronary artery, left anterior descending and left circumflex artery segments, respectively on CMRA were of diagnostic image quality (Figure 10) (66). The sensitivity, 

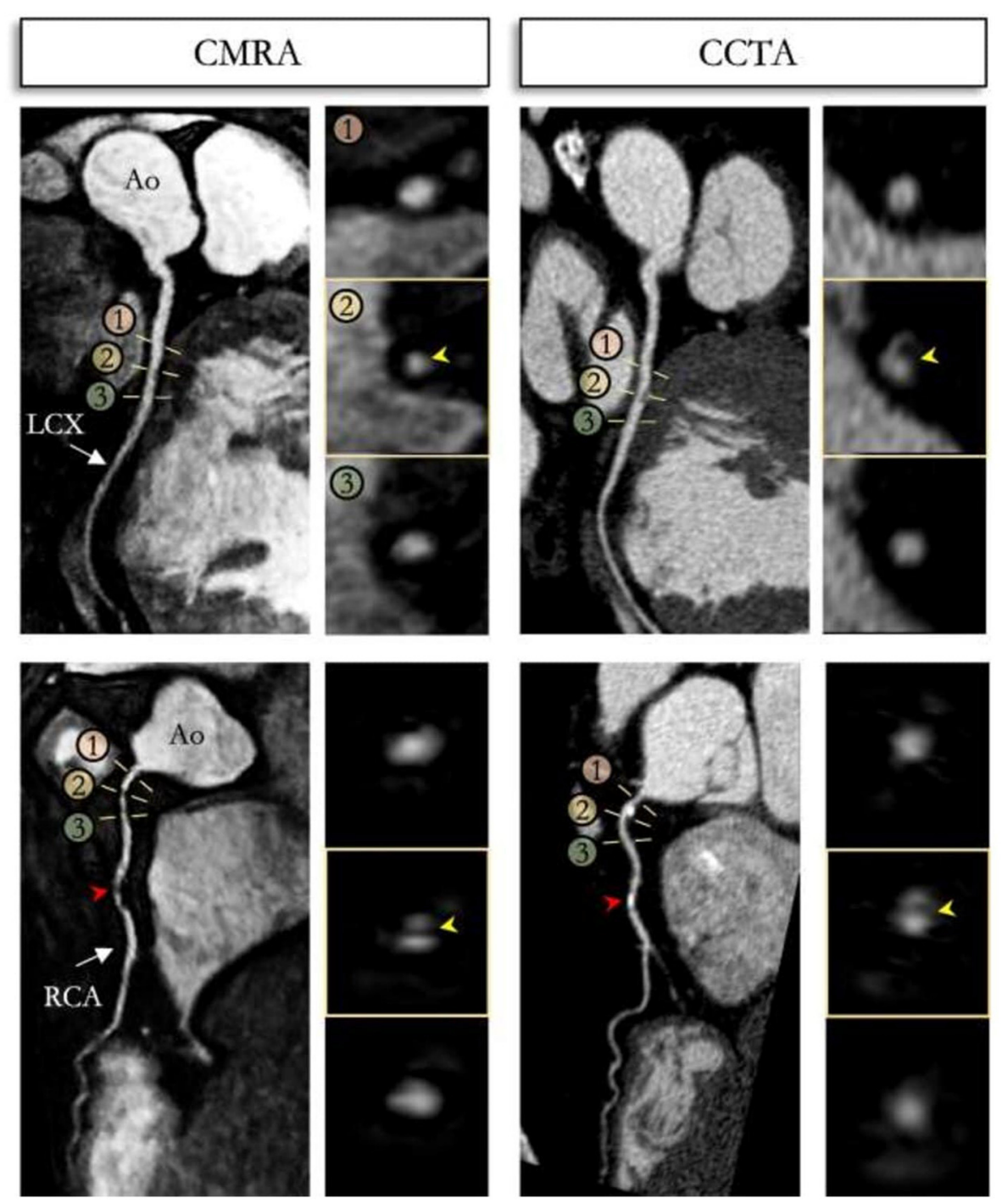

FIGURE 9 | Reformatted non-contrast whole-heart sub-millimeter isotropic CMRA (left) and CCTA (right) images along the LCX (top) and RCA (bottom) are shown for a 54 year-old male patient. The CMRA dataset was acquired in 9 min with 100\% scan efficiency (heart rate of 57 bpm). The CCTA images demonstrate mild (25-49\%) disease with a calcified plaque within the proximal RCA and severe disease (70-90\%) with a partially calcified plaque in the mid-segment of RCA (red arrows), and minimal (0-24\%) disease with calcified plaque in the mid-segment of the LCX. Luminal narrowing is seen on the cross-sectional views at the sites of coronary plaque on the CMRA images (yellow arrows). LAD, left anterior descending artery; RCA, right coronary artery; LCX, left circumflex artery; Ao, aorta. Adapted with permission from Bustin et al. (65).

specificity, positive predictive value, negative predictive value and diagnostic accuracy were as follows: per patient $(100,74,55,100$, and $80 \%)$, per vessel $(81,88,46,97$, and $88 \%)$ and per segment $(76,95,44,99$, and $94 \%)$, respectively, with an average acquisition time of $10.7 \mathrm{~min}$ at $0.9 \mathrm{~mm}$ isotropic spatial resolution (66).

Whilst an acquisition time of $\sim 10 \mathrm{~min}$ for sub- $1 \mathrm{~mm}$ isotropic spatial resolution is clinically feasible, it is nevertheless considerably longer than a full CCTA scan (even when coronary artery calcium scoring and contrast enhanced coronary angiography are combined). It is particularly disadvantageous if image quality is sub-optimal on first attempt and repeat imaging within the same scan session is required. Furthermore, reconstruction times of iterative methods are relatively long and computationally demanding, especially if combined with nonrigid motion correction or non-Cartesian trajectories. Finally, highly undersampled acquisitions are vulnerable to residual aliasing or staircasing and blurring artefacts, putting a realistic limit on the extend of conventional image acceleration methods. Recently, deep-learning based image reconstruction networks have been proposed to address some of these shortcomings (6771). The deep neural network scheme is trained to recognise prior reconstructed data samples retrospectively, which is then adapted to prospectively reconstruct acquired images in a matter of seconds (Figure 11) (72). However, whilst this scheme partially 


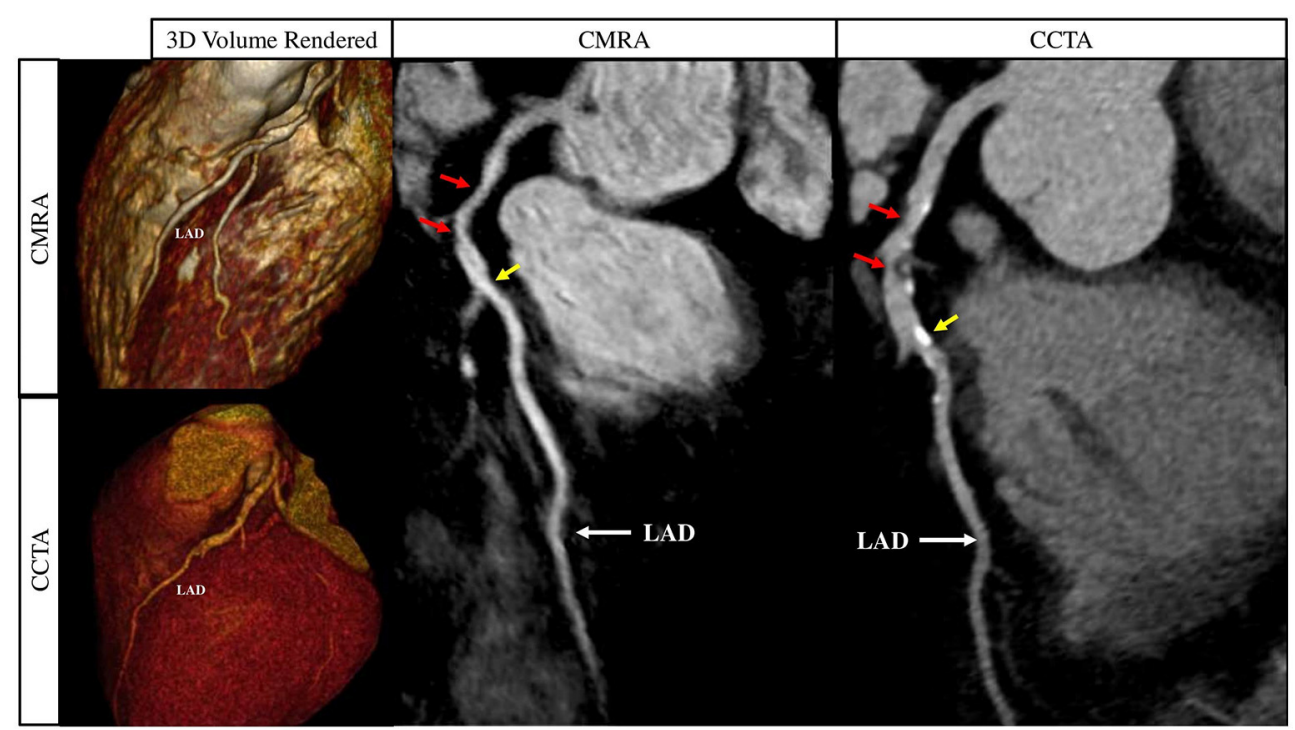

FIGURE 10 | Curved multiplanar reformat and 3D volume rendered non-contrast CMRA and contrast enhanced CCTA in a 60 year old male with $>50 \%$ partially calcified stenosis in the proximal to mid LAD on either side of the first diagonal artery (red arrows). The yellow arrows represent a focal calcified $<50 \%$ stenosis just distal to the second diagonal artery. CMRA, Coronary Magnetic Resonance Angiography; CCTA, Coronary Computed Tomography Angiography; LAD, Left Anterior Descending Artery. Adapted with permission from Hajhosseiny et al. (66).

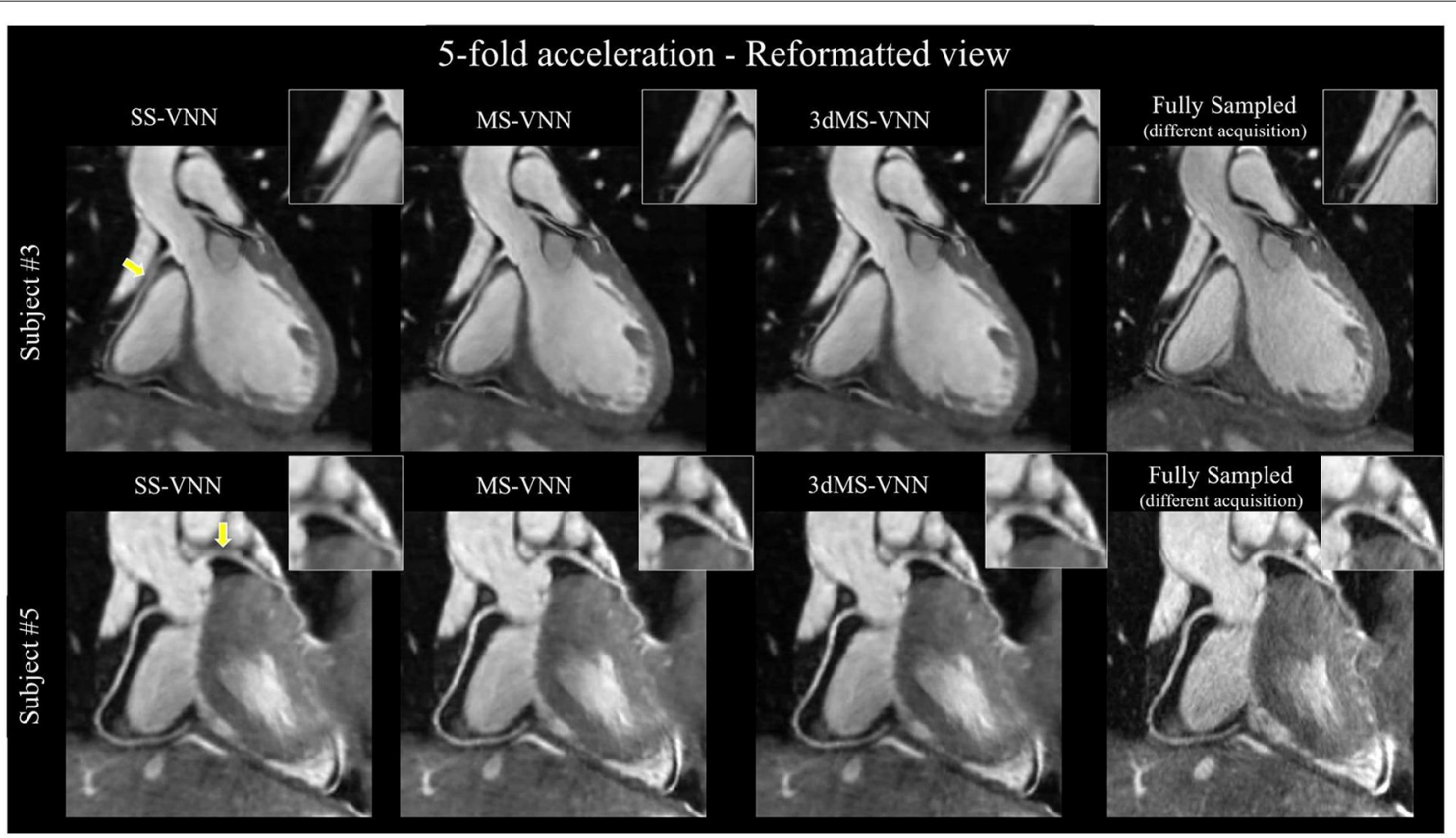

FIGURE 11 I CMRA images reconstructed with a single-scale VNN (SS-VNN), the multi-scale VNN (MS-VNN), and a pseudo 3D multi-scale VNN (3dMS-VNN). CMRA images were reformatted along the left (LAD) and right (RCA) coronary arteries, for two representative healthy subjects. Fully sampled and undersampled acquisitions with acceleration factor of $5 \times$ are shown. VNN, variational neural network. Adapted with permission from Fuin et al. (72). 


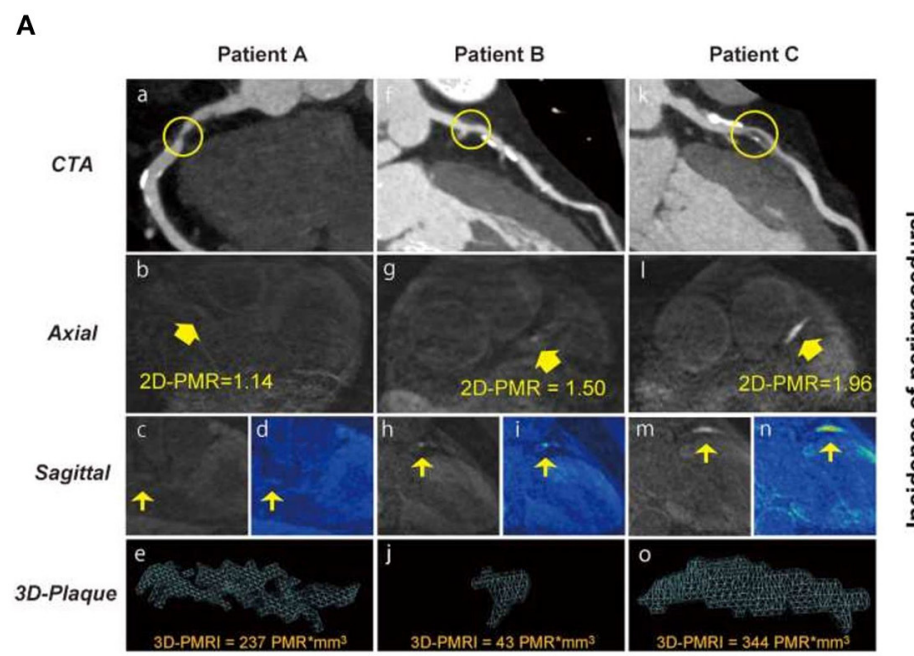

B

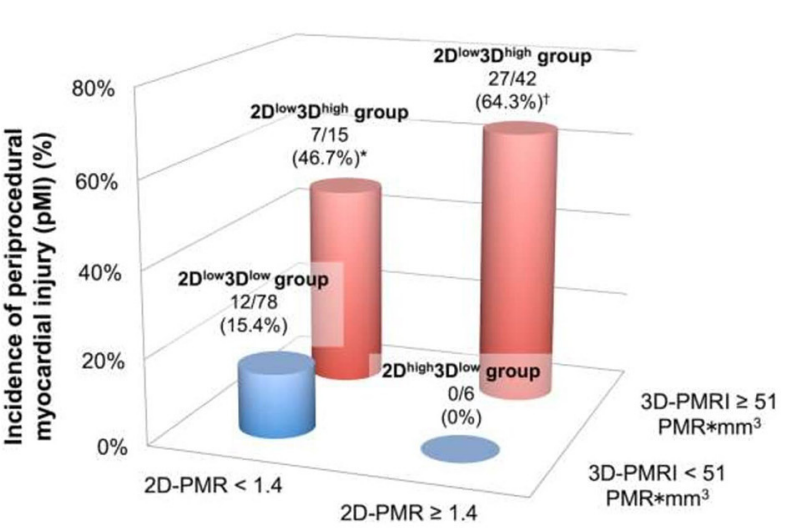

FIGURE 12 | (A) Representative 2-dimensional and 3-dimensional plaque assessment on T1-weighted imaging. Coronary plaques with $2 D^{\text {low } 3 D^{\text {high }}}$ in the proximal right coronary artery (2D-PMR, 1.14; 3Di-PMR, $237 \mathrm{PMR}^{*} \mathrm{~mm}^{3}$; Patient A: a-e), 2D high $3 \mathrm{D}^{\text {low }}$ in the proximal left anterior descending artery (LAD) (2D-PMR, 1.50; 3Di-PMR, 43 PMR ${ }^{*} \mathrm{~mm}^{3}$; Patient B: $\left.f-j\right)$, and 2D high $3 \mathrm{D}^{\text {high }}$ in the proximal LAD (2D-PMR, 1.96; 3Di-PMR, $344 \mathrm{PMR}^{\star} \mathrm{mm}^{3}$; Patient C: k-0). Computed tomography angiography (CTA) images $(a, f, k)$, and axial images $(b, g, l)$, sagittal images $(c, h, m)$, colour maps $(d, l, n)$, and 3D region of interests (3D plaque: e, j, n) on T1w images are shown. Yellow circles indicate percutaneous coronary intervention target lesion sites on CTA. Yellow arrows indicate lesions on T1w imaging corresponding to a lesion on angiography that underwent intervention. (B) Incidence of periprocedural myocardial injury (pMI) based on 3Di-PMR and 2D-PMR cutoff values. The red and blue bars represent patients with $3 \mathrm{Di}-\mathrm{PMR} \geq 51 \mathrm{PMR}^{*} \mathrm{~mm}^{3}$ and $<51 \mathrm{PMR}^{*} \mathrm{~mm}^{3}$, respectively. $P<0.001$ based on the chi-squared test. ${ }^{*} P=0.006$ vs. $2 D^{\text {high }} 3 D^{\text {low }}$ group. ${ }^{\dagger} P<0.001$ vs. $2 D^{\text {low } 3} 3 D^{\text {low }}$ group, and $P=0.003$ vs. $2 D^{\text {high }} 3 D^{\text {low }}$ group. Adapted with permission from Hosoda et al. (90).

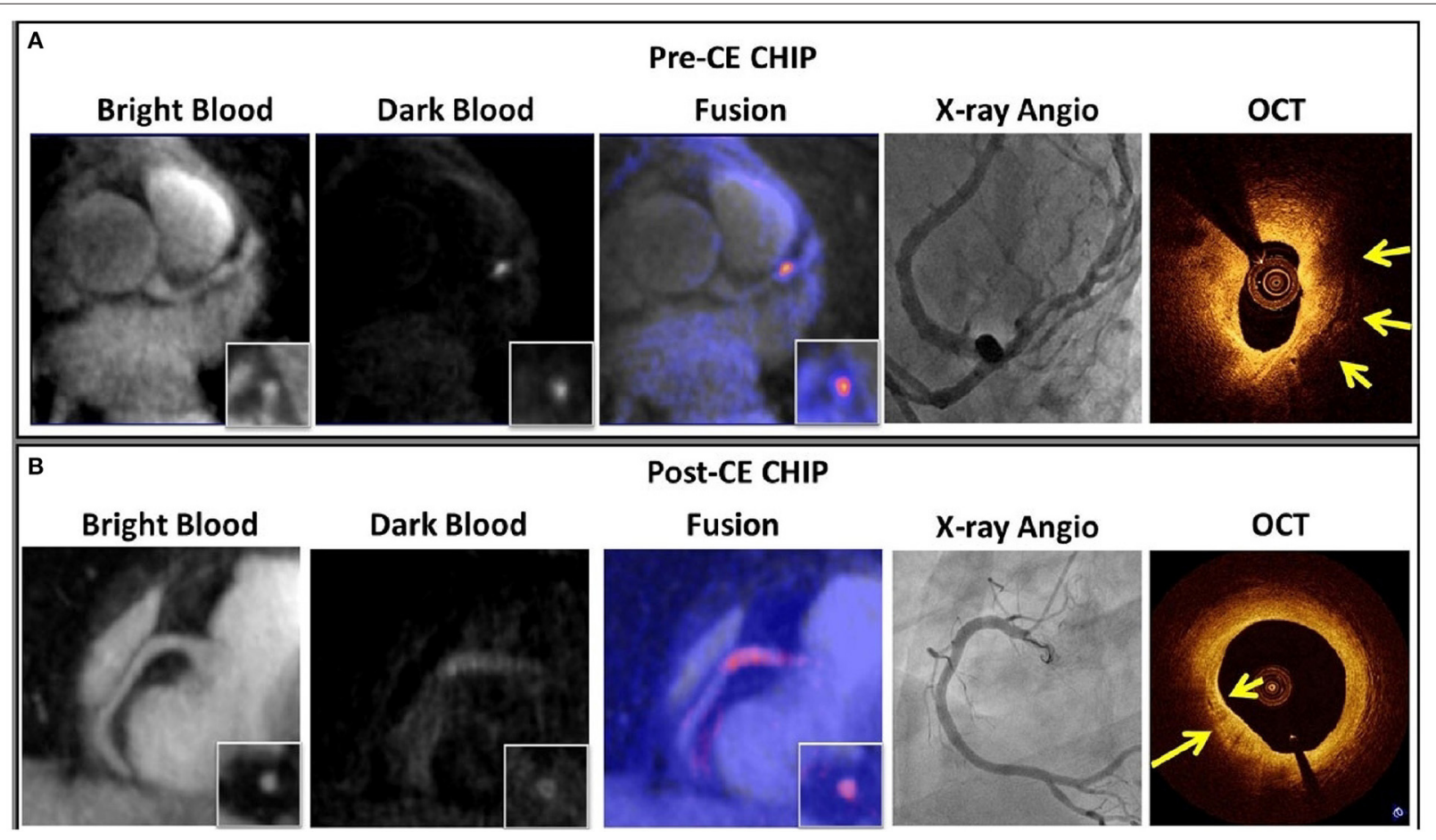

FIGURE 13 | (A) An example of pre-CE CHIP was found in the middle LAD visualised on the dark blood images and fused with the bright-blood scan. XA showed significant stenosis (70\%) at that location. OCT showed large signal-poor area suggestive of possible lipid core and/or intra-plaque haemorrhage (yellow arrow). (B) An example of post-CE CHIP with diffuse wall enhancement at proximal RCA as localized on the bright-blood images. XA showed only mild stenosis (30\%) at that location. OCT showed strong multi-focal back reflections and signal heterogeneity within the overlaying tissue suggestive of high macrophage density (yellow arrows). $\mathrm{CE}$, contrast enhancement; CHIP, coronary hyper-intensive plaques; XA, X-ray angiography; OCT, optical coherence tomography. Adapted with permission from Xie et al. (96). 

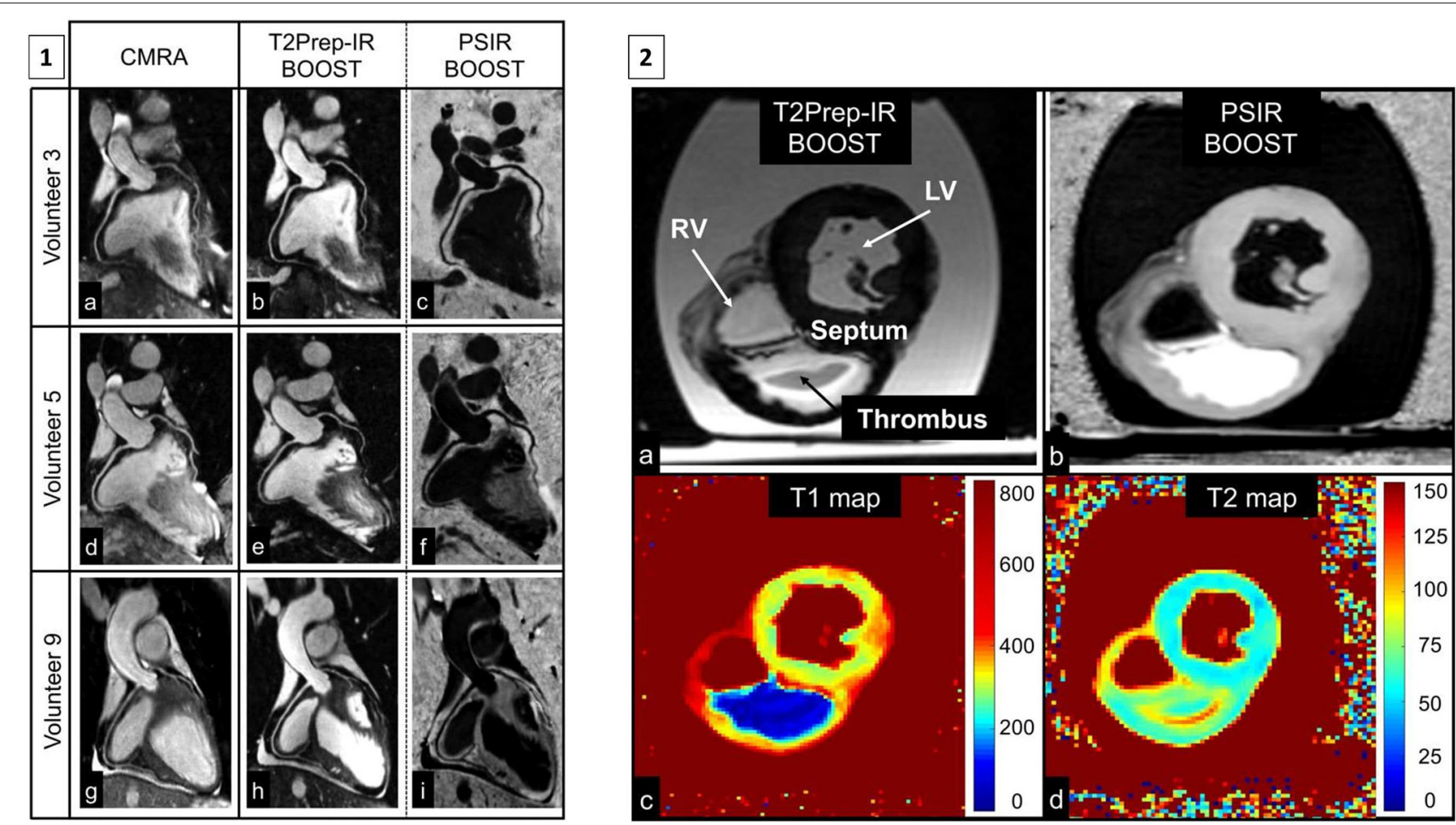

FIGURE 14 | (1) Reformatted coronary depiction in three representative healthy volunteers obtained with a conventional $\mathrm{T}_{2}$-prepared bright-blood CMRA acquisition ( $\mathrm{a}, \mathrm{d}, \mathrm{g}$ ) and the proposed BOOST sequence for simultaneous bright-blood ( $\mathrm{T}_{2}$ Prep-IR BOOST datasets in b, e, h) and black-blood (PSIR BOOST datasets in c, f, i)

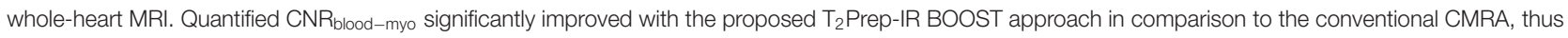
leading to a higher quantified coronary percentage vessel sharpness (\%VS) for both right and left coronary arteries. In the PSIR BOOST images in (c, f, i), the efficacy of blood signal suppression can be appreciated along multiple portions of the coronary tree. (2) MRI images obtained in the ex vivo pig heart. All the images depict a short-axis view at the midventricular level. Images acquired with the proposed BOOST sequence are reported in (a) (bright-blood $\mathrm{T}_{2}$ Prep-IR dataset) and in (b) (black-blood PSIR-like reconstruction). RV, LV, thrombus, and interventricular septum are indicated. The black-blood reconstruction (b) clearly enhances the signal from the thrombus when compared to the bright-blood dataset (a). Furthermore, $2 \mathrm{D} \mathrm{T}_{1}$ (c) and $\mathrm{T}_{2}$ (d) mapping techniques were acquired. The ex vivo thrombus is characterized by a relatively short $\mathrm{T}_{1}$ and $\mathrm{T}_{2}$. BOOST, Bright-blood and black-bIOOd phase SensiTive; IR, inversion recovery; myo, myocardium; PSIR,

phase-sensitive inversion recovery; $T_{2}$ Prep, $T_{2}$ prepared; LV, left ventricular cavity; RV, right ventricular cavity. Adapted with permission from Ginami et al. (98).

resolves the time-consuming image reconstruction process, it still relies on a prospectively acquired high spatial resolution CMRA acquisition. Recently, deep learning based super resolution schemes have been proposed to overcome this drawback. Here, a low spatial resolution image is prospectively acquired, often with low to intermediate acceleration factors, thereby significantly shortening the time the patient is on the scanning table, whilst simultaneously employing trained deep neural networks to reconstruct to a higher target spatial resolution within a few seconds (73-82). These novel deep learning schemes have the potential to finally unlock the true potential of CMRA in the clinical setting, which require further prospective clinical validation against CCTA and invasive X-ray angiography.

\section{MAGNETIC RESONANCE CORONARY PLAQUE IMAGING}

Our understanding of CAD and CCS is rapidly evolving. The COURAGE trial highlighted the limitations of coronary luminography as a standalone prognostic measure of CAD, with no significant difference in revascularisation vs. best medical therapy on hard end point outcomes such as death or myocardial infarction in patients that were angiographically identified to have significant CAD (83). The FAME trials brought into sharp focus the prognostic benefits of functional physiological assessment of CAD, demonstrating supplementary benefits in terms of death, myocardial infarction and urgent revascularisation when coronary intervention was based on functional assessment with FFR compared with invasive angiography alone or medical therapy alone $(7,84)$. However, in the recently published ISCHEMIA trial of 5,179 patients with CCS, there was no prognostic or indeed symptomatic benefit of functionally guided revascularisation vs. medical therapy alone after 4 years of follow up, although patients with left main stenosis were excluded from this study (85). Therefore, academic focus is on finding a more specific marker in patients with CCS who are more likely to have a prognostically worse clinical outcome (86-88). Identification of high-risk coronary plaques by CMR has been shown to be an independent and prognostically significant marker of CAD in patients with CCS, in addition to or indeed regardless of coronary luminal stenosis 
(89). It has also recently been shown to predict periprocedural myocardial injury (Figure 12) (90). CMR has the capability to detect vulnerable coronary plaques by taking advantage of the intrinsic T1 shortening of plaque components (e.g., intraplaque haemorrhage, thrombus, and lipid core), both with and without the need for contrast agents (91-95).

Despite this, MR coronary plaque imaging is constrained by comparable technical challenges to CMRA, e.g., respiratory motion, prohibitively long and unpredictable acquisition time, plaque to coronary artery misalignment due to the sequential nature of data acquisition. Recently, a novel framework (CATCH) has been proposed to overcome some of these limitations for MR coronary plaque imaging. This framework assembles advanced motion correction techniques outlined above to facilitate simultaneous multi contrast bright and black blood coronary artery angiography and visualisation of vulnerable coronary plaque (Figure 13) (96, 97). However, there is incomplete overlap of respiratory motion parameters between the bright-blood and black-blood datasets, potentially resulting in residual coronary plaque-misregistration. More recently, another framework has been proposed (BOOST) for simultaneous coronary angiography and thrombus/intraplaque haemorrhage visualisation (Figure 14) (98). This framework works by acquiring two differently weighted bright-blood datasets in alternate heart beats, which are subsequently processed in a phase-sensitive inversion recovery (PSIR)-like reconstruction to produce a third, black-blood dataset. The two bright-blood datasets enable respiratory motion to be independently estimated and corrected, which reduces the likelihood of misregistration artefacts. It also uses the iNAV technology with a highly undersampled golden angle Cartesian acquisition, which enables $100 \%$ respiratory scan efficiency, 2D rigid translational motion correction as well as $3 \mathrm{D}$ non-rigid motion estimation. Traditional reconstruction frameworks (e.g., iterative sense, compressed sensing or low rank patched based frameworks) or deep learning neural network reconstruction can be applied. These frameworks require further extensive clinical validation, ideally against intravascular imaging modalities such as intravascular ultrasound or optical coherence tomography.

It is also possible to directly target specific components of the atherosclerotic plaque (99-101). Various MR specific contrast agents are currently in development which can target molecular components that are involved in atherosclerotic plaque initiation, progression, instability and plaque rupture, such as elastin (102), tropoelastin, collagen $(103,104)$, fibrin (105), and matrix

\section{REFERENCES}

1. Virani SS, Alonso A, Benjamin EJ, Bittencourt MS, Callaway CW, Carson AP, et al. Heart disease and stroke statistics-2020 update: a report from the American Heart Association. Circulation. (2020) 141:e33. doi: 10.1161/CIR.00000000000 00746

2. Institute for Health Metrics and Evaluation (IHME). GBD Compare. Available online at: https://vizhub.healthdata.org/gbd-compare/ (accessed June 6, 2021). metalloproteinases (106). Whilst these frameworks have shown great promise in pre-clinical feasibility studies, the challenge is to safely replicate these findings in clinical validation studies.

\section{CONCLUSIONS}

Chronic coronary syndrome is a progressive and multifaceted condition associated with coronary artery atherosclerosis, which manifests clinically as either angina, heart failure and gradual left ventricular dysfunction, arrhythmia and/or acute myocardial infarction. The timely recognition of CCS enables bespoke risk assessment of patients, prompt initiation of therapeutical intervention and long-term monitoring of potential complications. Amongst a maelstrom of diagnostic imaging modalities for CAD, CMRA could potentially emerge as a viable, versatile, reproducible and non-invasive imaging modality for the assessment of CCS, which is free of ionising radiation, iodinated contrast agents and can be performed without gadolinium contrast enhancement. These characteristics would be ideally suited for repeat imaging of patients to monitor disease progression and gauge response to treatment. Furthermore, it can be combined with myocardial volumetric assessment, tissue characterisation, perfusion and coronary plaque assessment to enable comprehensive assessment of ischaemic heart disease.

\section{AUTHOR CONTRIBUTIONS}

RH drafting and editing of the manuscript. CM, GC, RK, WK, $\mathrm{CP}$, and $\mathrm{RB}$ reviewing and editing of manuscript. All authors contributed to the article and approved the submitted version.

\section{FUNDING}

This work was supported by the following grants: (1) EPSRC $\mathrm{EP} / \mathrm{P} 032311 / 1, \mathrm{EP} / \mathrm{P} 001009 / 1$, and EP/P007619/1, (2) BHF programme grant $\mathrm{RG} / 20 / 1 / 34802$, (3) King's BHF Centre for Research Excellence RE/18/2/34213, (4) Wellcome EPSRC Centre for Medical Engineering (NS/A000049/1), and (5) Department of Health through the National Institute for Health Research (NIHR) comprehensive Biomedical Research Centre award to Guy's \& St Thomas' NHS Foundation Trust in partnership with King's College London and King's College Hospital NHS Foundation Trust and by the NIHR MedTech Cooperative for Cardiovascular Disease at Guy's and St Thomas' NHS Foundation Trust. 
management of chronic coronary syndromes. Eur Heart J. (2020) 41:407-77. doi: 10.1093/eurheartj/ehz425

7. De Bruyne B, Pijls NHJ, Kalesan B, Barbato E, Tonino PAL, Piroth Z, et al. Fractional flow reserve-guided PCI versus medical therapy in stable coronary disease. N Engl J Med. (2012) 367:991-1001. doi: 10.1056/NEJMoa1205361

8. Götberg $\mathrm{M}$, Christiansen $\mathrm{EH}$, Gudmundsdottir IJ, Sandhall L, Danielewicz M, Jakobsen $\mathrm{L}$, et al. Instantaneous wave-free ratio versus fractional flow reserve to guide PCI. N Engl J Med. (2017) 376:1813-23. doi: 10.1056/NEJMoa1616540

9. Rathod KS, Hamshere SM, Jones DA, Mathur A. Intravascular ultrasound versus optical coherence tomography for coronary artery imaging - apples and oranges? Interv Cardiol Rev. (2015) 10:8-15. doi: 10.15420/icr.2015.10.1.8

10. Tian J, Dauerman H, Toma C, Samady H, Itoh T, Kuramitsu S, et al. Prevalence and characteristics of TCFA and degree of coronary artery stenosis. J Am Coll Cardiol. (2014) 64:672-80. doi: 10.1016/j.jacc.2014.05.052

11. Arakelyan K, Cantow K, Hentschel J, Flemming B, Pohlmann A, Ladwig M, et al. Early effects of an $\mathrm{x}$-ray contrast medium on renal $\mathrm{T}(2) * / \mathrm{T}(2) \mathrm{MRI}$ as compared to short-term hyperoxia, hypoxia and aortic occlusion in rats. Acta Physiol. (2013) 208:202-13. doi: 10.1111/apha.12094

12. Kolossváry M, Szilveszter B, Merkely B, Maurovich-Horvat P. Plaque imaging with CT-a comprehensive review on coronary CT angiography based risk assessment. Cardiovasc Diagn Ther. (2017) 7:489-506. doi: 10.21037/cdt.2016.11.06

13. Non invasive Coronary Imaging With Computed Tomography. Available online at: https://www.escardio.org/Journals/E-Journal-of-CardiologyPractice/Volume-5/Non-Invasive-Coronary-Imaging-With-ComputedTomography-Title-Non-Invasive-Cor (accessed August 14, 2018).

14. Alfakih K, Byrne J, Monaghan M. CT coronary angiography: a paradigm shift for functional imaging tests. Open Hear. (2018) 5:e000754. doi: 10.1136/openhrt-2017-000754

15. Doris MK, Newby DE. How should CT coronary angiography be integrated into the management of patients with chest pain and how does this affect outcomes? Eur Hear J Qual Care Clin Outcomes. (2016) 2:7280. doi: 10.1093/ehjqcco/qcv027

16. Kim WY, Danias PG, Stuber M, Flamm SD, Plein S, Nagel E, et al. Coronary magnetic resonance angiography for the detection of coronary stenoses. $N$ Engl J Med. (2001) 345:1863-69. doi: 10.1056/NEJMoa010866

17. Kato S, Kitagawa K, Ishida N, Ishida M, Nagata M, Ichikawa Y, et al. Assessment of coronary artery disease using magnetic resonance coronary angiography: a national multicenter trial. J Am Coll Cardiol. (2010) 56:98391. doi: 10.1016/j.jacc.2010.01.071

18. Hamdan A, Asbach P, Wellnhofer E, Klein C, Gebker R, Kelle S, et al. A prospective study for comparison of MR and CT imaging for detection of coronary artery stenosis. JACC Cardiovasc Imaging. (2011) 4:50-61. doi: 10.1016/j.jcmg.2010.10.007

19. Hamdan A, Doltra A, Huppertz A, Wellnhofer E, Fleck E, Kelle S. Comparison of coronary magnetic resonance and computed tomography angiography for prediction of cardiovascular events. JACC Cardiovasc Imaging. (2014) 7:1063-65. doi: 10.1016/j.jcmg.2014.03.020

20. Yoon YE, Kitagawa K, Kato S, Ishida M, Nakajima H, Kurita T, et al. Prognostic value of coronary magnetic resonance angiography for prediction of cardiac events in patients with suspected coronary artery disease. J Am Coll Cardiol. (2012) 60:2316-22. doi: 10.1016/j.jacc.2012.07.060

21. Coppo S, Piccini D, Bonanno G, Chaptinel J, Vincenti G, Feliciano H, et al. Free-running 4D whole-heart self-navigated golden angle MRI: initial results. Magn Reson Med. (2015) 74:1306-16. doi: 10.1002/mrm.25523

22. Pang J, Bhat H, Sharif B, Fan Z, Thomson LEJ, LaBounty T, et al. Whole-heart coronary MRA with $100 \%$ respiratory gating efficiency: self-navigated threedimensional retrospective image-based motion correction (TRIM). Magn Reson Med. (2014) 71:67-74. doi: 10.1002/mrm.24628

23. Atkinson DJ, Edelman RR. Cineangiography of the heart in a single breath hold with a segmented turboFLASH sequence. Radiology. (1991) 178:35760. doi: 10.1148/radiology.178.2.1987592

24. Edelman RR, Manning WJ, Burstein D, Paulin S. Coronary arteries: breath-hold MR angiography. Radiology. (1991) 181:641-3. doi: 10.1148/radiology.181.3.1947074
25. Meyer CH, Hu BS, Nishimura DG, Macovski A. Fast spiral coronary artery imaging. Magn Reson Med. (1992) 28:202-13. doi: 10.1002/mrm.1910280204

26. Manning WJ, Li W, Edelman RR. A preliminary report comparing magnetic resonance coronary angiography with conventional angiography. $\mathrm{N} \mathrm{Engl} \mathrm{J}$ Med. (1993) 328:828-32. doi: 10.1056/NEJM199303253281202

27. Wielopolski PA, van Geuns RJ, de Feyter PJ, Oudkerk M. Breath-hold coronary MR angiography with volume-targeted imaging. Radiology. (1998) 209:209-19. doi: 10.1148/radiology.209.1.9769834

28. Li D, Carr JC, Shea SM, Zheng J, Deshpande VS, Wielopolski PA, et al. Coronary arteries: magnetization-prepared contrast-enhanced threedimensional volume-targeted breath-hold MR angiography. Radiology. (2001) 219:270-7. doi: 10.1148/radiology.219.1.r01ap37270

29. Niendorf T, Hardy CJ, Giaquinto RO, Gross P, Cline HE, Zhu Y, et al. Toward single breath-hold whole-heart coverage coronary MRA using highly accelerated parallel imaging with a 32-channel MR system. Magn Reson Med. (2006) 56:167-76. doi: 10.1002/mrm.20923

30. Li D, Kaushikkar S, Haacke EM, Woodard PK, Dhawale PJ, Kroeker RM, et al. Coronary arteries: three-dimensional MR imaging with retrospective respiratory gating. Radiology. (1996) 201:857-63. doi: 10.1148/radiology.201.3.8939242

31. Danias PG, Stuber M, Botnar RM, Kissinger K V, Chuang ML, Manning WJ. Navigator assessment of breath-hold duration: impact of supplemental oxygen and hyperventilation. Am J Roentgenol. (1998) 171:395-7. doi: 10.2214/ajr.171.2.9694460

32. Botnar RM, Stuber M, Danias PG, Kissinger K V, Manning WJ. Improved coronary artery definition with T2-weighted, freebreathing, three-dimensional coronary MRA. Circulation. (1999) 99:3139-48. doi: 10.1161/01.CIR.99.24.3139

33. Runge VM, Clanton JA, Partain CL, James AE. Respiratory gating in magnetic resonance imaging at 0.5 Tesla. Radiology. (1984) 151:5213. doi: 10.1148/radiology.151.2.6709928

34. Felblinger J, Boesch C. Amplitude demodulation of the electrocardiogram signal (ECG) for respiration monitoring and compensation during MR examinations. Magn Reson Med. (1997) 38:129-36. doi: 10.1002/mrm.1910380118

35. Ehman RL, Felmlee JP. Adaptive technique for high-definition MR imaging of moving structures. Radiology. (1989) 173:25563. doi: 10.1148/radiology.173.1.2781017

36. McConnell M V, Khasgiwala VC, Savord BJ, Chen MH, Chuang ML, Edelman RR, et al. Comparison of respiratory suppression methods and navigator locations for MR coronary angiography. Am J Roentgenol. (1997) 168:1369-75. doi: 10.2214/ajr.168.5.9129447

37. Nehrke K, Börnert P, Manke D, Böck JC. Free-breathing cardiac MR imaging: study of implications of respiratory motion-initial results. Radiology. (2001) 220:810-5. doi: 10.1148/radiol.2203010132

38. Wang Y, Riederer SJ, Ehman RL. Respiratory motion of the heart: kinematics and the implications for the spatial resolution in coronary imaging. Magn Reson Med. (1995) 33:713-9. doi: 10.1002/mrm.1910330517

39. Nagel E, Bornstedt A, Schnackenburg B, Hug J, Oswald H, Fleck E. Optimization of realtime adaptive navigator correction for 3D magnetic resonance coronary angiography. Magn Reson Med. (1999) 42:408-11.

40. Stehning C, Börnert P, Nehrke K, Eggers H, Stuber M. Freebreathing whole-heart coronary MRA with 3D radial SSFP and self-navigated image reconstruction. Magn Reson Med. (2005) 54:476-80. doi: 10.1002/mrm.20557

41. Piccini D, Littmann A, Nielles-Vallespin S, Zenge MO. Respiratory selfnavigation for whole-heart bright-blood coronary MRI: methods for robust isolation and automatic segmentation of the blood pool. Magn Reson Med. (2012) 68:571-9. doi: 10.1002/mrm.23247

42. Lai P, Bi X, Jerecic R, Li D. A respiratory self-gating technique with $3 \mathrm{D}$ translation compensation for free-breathing whole-heart coronary MRA. Magn Reson Med. (2009) 62:731-8. doi: 10.1002/mrm.22058

43. Jhooti P, Gatehouse PD, Keegan J, Bunce NH, Taylor AM, Firmin DN. Phase ordering with automatic window selection (PAWS): a novel motion- resistant technique for 3D coronary imaging. Magn Reson Med. (2000) 43:47080. doi: 10.1002/(SICI)1522-2594(200003)43:3\&lt;470::AID-MRM20\&gt;3.0. $\mathrm{CO} ; 2-\mathrm{U}$ 
44. Keegan J, Jhooti P, Babu-Narayan S V., Drivas P, Ernst S, Firmin DN. Improved respiratory efficiency of $3 \mathrm{D}$ late gadolinium enhancement imaging using the continuously adaptive windowing strategy (CLAWS). Magn Reson Med. (2014) 71:1064-74. doi: 10.1002/mrm.24758

45. Jhooti P, Wiesmann F, Taylor AM, Gatehouse PD, Yang GZ, Keegan J, et al. Hybrid ordered phase encoding (HOPE): an improved approach for respiratory artifact reduction. J Magn Reson Imaging. (1998) 8:96880. doi: 10.1002/jmri.1880080428

46. Piccini D, Monney P, Sierro C, Coppo S, Bonanno G, van Heeswijk RB, et al. Respiratory self-navigated postcontrast whole-heart coronary MR angiography: initial experience in patients. Radiology. (2014) 270:37886. doi: 10.1148/radiol.13132045

47. Henningsson M, Koken P, Stehning C, Razavi R, Prieto C, Botnar RM. Whole-heart coronary MR angiography with 2D self-navigated image reconstruction. Magn Reson Med. (2012) 67:437-45. doi: 10.1002/mrm.23027

48. Kawaji K, Spincemaille P, Nguyen TD, Thimmappa N, Cooper MA, Prince $\mathrm{MR}$, et al. Direct coronary motion extraction from a 2D fat image navigator for prospectively gated coronary MR angiography. Magn Reson Med. (2014) 71:599-607. doi: 10.1002/mrm.24698

49. Scott AD, Keegan J, Firmin DN. Beat-to-beat respiratory motion correction with near 100\% efficiency: a quantitative assessment using highresolution coronary artery imaging. Magn Reson Imaging. (2011) 29:56878. doi: 10.1016/j.mri.2010.11.004

50. Wu HH, Gurney PT, Hu BS, Nishimura DG, McConnell M V. Freebreathing multiphase whole-heart coronary MR angiography using imagebased navigators and three-dimensional cones imaging. Magn Reson Med. (2013) 69:1083-93. doi: 10.1002/mrm.24346

51. Addy NO, Ingle RR, Luo J, Baron CA, Yang PC, Hu BS, Nishimura DG. 3D image-based navigators for coronary MR angiography. Magn Reson Med. (2017) 77:1874-83. doi: 10.1002/mrm.26269

52. Henningsson M, Shome J, Bratis K, Vieira MS, Nagel E, Botnar RM. Diagnostic performance of image navigated coronary CMR angiography in patients with coronary artery disease. J Cardiovasc Magn Reson. (2017) 19:68. doi: 10.1186/s12968-017-0381-3

53. Bhat H, Ge L, Nielles-Vallespin S, Zuehlsdorff S, Li D. 3D radial sampling and $3 \mathrm{D}$ affine transform-based respiratory motion correction technique for freebreathing whole-heart coronary MRA with 100\% imaging efficiency. Magn Reson Med. (2011) 65:1269-77. doi: 10.1002/mrm.22717

54. Piccini D, Feng L, Bonanno G, Coppo S, Yerly J, Lim RP, et al. Four-dimensional respiratory motion-resolved whole heart coronary $\mathrm{MR}$ angiography. Magn Reson Med. (2017) 77:1473-84. doi: 10.1002/mrm.26221

55. Feng L, Coppo S, Piccini D, Yerly J, Lim RP, Masci PG, et al. 5D whole-heart sparse MRI. Magn Reson Med. (2018) 79:826-38. doi: 10.1002/mrm.26745

56. Pang J, Chen Y, Fan Z, Nguyen C, Yang Q, Xie Y, et al. High efficiency coronary MR angiography with nonrigid cardiac motion correction. Magn Reson Med. (2016) 76:1345-53. doi: 10.1002/mrm.26332

57. Di Sopra L, Piccini D, Coppo S, Stuber M, Yerly J. An automated approach to fully self-gated free-running cardiac and respiratory motionresolved 5D whole-heart MRI. Magn Reson Med. (2019) 82:211832. doi: $10.1002 / \mathrm{mrm} .27898$

58. Addy NO, Ingle RR, Wu HH, Hu BS, Nishimura DG. High-resolution variable-density 3D cones coronary MRA. Magn Reson Med. (2015) 74:61421. doi: $10.1002 / \mathrm{mrm} .25803$

59. Malavé MO, Baron CA, Addy NO, Cheng JY, Yang PC, Hu BS, et al. Wholeheart coronary MR angiography using a 3D cones phyllotaxis trajectory. Magn Reson Med. (2019) 81:1092-103. doi: 10.1002/mrm.27475

60. Malavé MO, Baron CA, Koundinyan SP, Sandino CM, Ong F, Cheng JY, et al. Reconstruction of undersampled 3D non-Cartesian image-based navigators for coronary MRA using an unrolled deep learning model. Magn Reson Med. (2020) 84:800-12. doi: 10.1002/mrm.28177

61. Prieto C, Doneva M, Usman M, Henningsson M, Greil G, Schaeffter T, et al. Highly efficient respiratory motion compensated free-breathing coronary mra using golden-step Cartesian acquisition. J Magn Reson Imaging. (2015) 41:738-46. doi: 10.1002/jmri.24602

62. Cruz G, Atkinson D, Henningsson M, Botnar RM, Prieto C. Highly efficient nonrigid motion-corrected $3 \mathrm{D}$ whole-heart coronary vessel wall imaging. Magn Reson Med. (2017) 77:1894-908. doi: 10.1002/mrm.26274
63. Bustin A, Ginami G, Cruz G, Correia T, Ismail TF, Rashid I, et al. Five-minute whole-heart coronary MRA with sub-millimeter isotropic resolution, 100\% respiratory scan efficiency, and 3D-PROST reconstruction. Magn Reson Med. (2019) 81:102-15. doi: 10.1002/mrm.27354

64. Akçakaya M, Basha TA, Goddu B, Goepfert LA, Kissinger K V., Tarokh V, et al. Low-dimensional-structure self-learning and thresholding: regularization beyond compressed sensing for MRI reconstruction. Magn Reson Med. (2011) 66:756-67. doi: 10.1002/mrm.22841

65. Bustin A, Rashid I, Cruz G, Hajhosseiny R, Correia T, Neji R, et al. 3D whole-heart isotropic sub-millimeter resolution coronary magnetic resonance angiography with non-rigid motion-compensated PROST. J Cardiovasc Magn Reson. (2020) 22:24. doi: 10.1186/s12968-02000611-5

66. Hajhosseiny R, Rashid I, Bustin A, Munoz C, Cruz G, Nazir MS, et al. Clinical comparison of sub-mm high-resolution non-contrast coronary CMR angiography against coronary CT angiography in patients with lowintermediate risk of coronary artery disease: a single center trial. J Cardiovasc Magn Reson. (2021) 23:57. doi: 10.1186/s12968-021-00758-9

67. Hyun CM, Kim HP, Lee SM, Lee S, Seo JK. Deep learning for undersampled MRI reconstruction. Phys Med Biol. (2018) 63:135007. doi: 10.1088/1361-6560/aac71a

68. Lin DJ, Johnson PM, Knoll F, Lui YW. Artificial intelligence for mr image reconstruction: an overview for clinicians. J Magn Reson Imaging. (2020) 53:1015-1028. doi: 10.1002/jmri.27078

69. Lundervold AS, Lundervold A. An overview of deep learning in medical imaging focusing on MRI. Z Med Phys. (2019) 29:10227. doi: 10.1016/j.zemedi.2018.11.002

70. Hammernik K, Knoll F. Machine learning for image reconstruction. In: Zhou SK, Rueckert D, Fichtinger G, editors. Handbook of Medical Image Computing and Computer Assisted Intervention. London; San Diego, CA; Cambridge; Oxford: Elsevier (2020). p. 25-64.

71. Bustin A, Fuin N, Botnar RM, Prieto C. From compressed-sensing to artificial intelligence-based cardiac MRI reconstruction. Front Cardiovasc Med. (2020) 7:17. doi: 10.3389/fcrm.2020.00017

72. Fuin N, Bustin A, Küstner T, Oksuz I, Clough J, King AP, et al. A multi-scale variational neural network for accelerating motion-compensated wholeheart 3D coronary MR angiography. Magn Reson Imaging. (2020) 70:15567. doi: 10.1016/j.mri.2020.04.007

73. Glasner D, Bagon S, Irani M. Super-resolution from a single image. In: Proceedings of the IEEE International Conference on Computer Vision. Kyoto (2009). p. 349-356.

74. Chen Y, Christodoulou AG, Zhou Z, Shi F, Xie Y, Li D. MRI superresolution with GAN and 3D multi-level densenet: smaller, faster, and better. arXiv. (2020).

75. Shi J, Liu Q, Wang C, Zhang Q, Ying S, Xu H. Super-resolution reconstruction of MR image with a novel residual learning network algorithm. Phys Med Biol. (2018) 63:085011. doi: 10.1088/1361-6560/aab9e9

76. Pham C-H, Tor-Díez C, Meunier H, Bednarek N, Fablet R, Passat $\mathrm{N}$, et al. Multiscale brain MRI super-resolution using deep 3D convolutional networks. Comput Med Imaging Graph. (2019) 77:101647. doi: 10.1016/j.compmedimag.2019.101647

77. Küstner T, Fuin N, Hammernik K, Bustin A, Qi H, Hajhosseiny R, et al. CINENet: deep learning-based 3D cardiac CINE MRI reconstruction with multi-coil complex-valued 4D spatio-temporal convolutions. Sci Rep. (2020) 10:13710. doi: 10.1038/s41598-020-70551-8

78. Steeden JA, Quail M, Gotschy A, Mortensen KH, Hauptmann A, Arridge $\mathrm{S}$, et al. Rapid whole-heart CMR with single volume super-resolution. $J$ Cardiovasc Magn Reson. (2020) 22:56. doi: 10.1186/s12968-020-00651-x

79. Xie Y, Lin R, Chen Y, Zhang Y, Shi F, Fei Y, et al. Super resolution $\{\mathrm{MRI}\}$ using 3D generative adversarial network: towards single breath-hold coronary $\{\mathrm{MR}\}$ angiography. In: Proceedings of the Joint Annual Meeting ISMRM-ESMRMB. Paris (2018).

80. Qi H, Cruz G, Kuestner T, Botner RM, Prieto C. Deep-learning based super-resolution reconstruction for sub-millimeter 3D isotropic coronary MR angiography in less than a minute. In: SMRA Virtual Meeting. (2020).

81. Küstner T, Fuin N, Bustin A, Qi H, Munoz C, Neji R, et al. Deep-learning based super-resolution reconstruction for sub-millimeter 3D isotropic coronary MR angiography. In: ESMRMB Virtual Meeting. (2020). 
82. Chen Y, Kwan A, Yang Q, Christodoulou AG, Li DYX. Ultra-fast coronary MRA by generative adversarial super-resolution. In: SMRA, Abstract \#16 Virtual Meeting. (2020).

83. Boden WE, O’Rourke RA, Teo KK, Hartigan PM, Maron DJ, Kostuk WJ, et al. Optimal medical therapy with or without PCI for stable coronary disease. N Engl J Med. (2007) 356:1503-16. doi: 10.1056/NEJMoa070829

84. Tonino PAL, De Bruyne B, Pijls NHJ, Siebert U, Ikeno F, van ' $t$ Veer $\mathrm{M}$, et al. Fractional flow reserve versus angiography for guiding percutaneous coronary intervention. N Engl J Med. (2009) 360:21324. doi: 10.1056/NEJMoa0807611

85. Maron DJ, Hochman JS, Reynolds HR, Bangalore S, O’Brien SM, Boden WE, et al. Initial invasive or conservative strategy for stable coronary disease. $N$ Engl J Med. (2020) 382:1395-407. doi: 10.1056/NEJMoa1915922

86. Tomaniak M, Katagiri Y, Modolo R, de Silva R, Khamis RY, Bourantas C V, et al. Vulnerable plaques and patients: state-of-the-art. Eur Heart J. (2020) 41:2997-3004. doi: 10.1093/eurheartj/ehaa227

87. van den Berg VJ, Haskard DO, Fedorowski A, Hartley A, Kardys I, Caga-Anan M, et al. IgM anti-malondialdehyde low density lipoprotein antibody levels indicate coronary heart disease and necrotic core characteristics in the Nordic Diltiazem (NORDIL) study and the Integrated Imaging and Biomarker Study 3 (IBIS-3). EBioMedicine. (2018) 36:6372. doi: 10.1016/j.ebiom.2018.08.023

88. Abdelrahman KM, Chen MY, Dey AK, Virmani R, Finn A V, Khamis RY, et al. Coronary computed tomography angiography from clinical uses to emerging technologies: JACC state-of-the-art review. J Am Coll Cardiol. (2020) 76:1226-43. doi: 10.1016/j.jacc.2020.06.076

89. Noguchi T, Kawasaki T, Tanaka A, Yasuda S, Goto Y, Ishihara M, et al. Highintensity signals in coronary plaques on noncontrast T1-weighted magnetic resonance imaging as a novel determinant of coronary events. J Am Coll Cardiol. (2014) 63:989-99. doi: 10.1016/j.jacc.2013.11.034

90. Hosoda H, Asaumi Y, Noguchi T, Morita Y, Kataoka Y, Otsuka $\mathrm{F}$, et al. Three-dimensional assessment of coronary high-intensity plaques with T1-weighted cardiovascular magnetic resonance imaging to predict periprocedural myocardial injury after elective percutaneous coronary intervention. J Cardiovasc Magn Reson. (2020) 22:5. doi: 10.1186/s12968-019-0588-6

91. Matsumoto K, Ehara S, Hasegawa T, Sakaguchi M, Otsuka K, Yoshikawa J, et al. Localization of coronary high-intensity signals on T1-weighted MR imaging: relation to plaque morphology and clinical severity of angina pectoris. JACC Cardiovasc Imaging. (2015) 8:1143-52. doi: 10.1016/j.jcmg.2015.06.013

92. Ehara S, Hasegawa T, Nakata S, Matsumoto K, Nishimura S, Iguchi T, et al. Hyperintense plaque identified by magnetic resonance imaging relates to intracoronary thrombus as detected by optical coherence tomography in patients with angina pectoris. Eur Hear J Cardiovasc Imaging. (2012) 13:394-9. doi: 10.1093/ehjci/jer305

93. Maintz D, Ozgun M, Hoffmeier A, Fischbach R, Kim WY, Stuber M, et al. Selective coronary artery plaque visualization and differentiation by contrast-enhanced inversion prepared MRI. Eur Heart J. (2006) 27:17326. doi: 10.1093/eurheartj/ehl102

94. Yeon SB, Sabir A, Clouse M, Martinezclark PO, Peters DC, Hauser $\mathrm{TH}$, et al. Delayed-enhancement cardiovascular magnetic resonance coronary artery wall imaging. J Am Coll Cardiol. (2007) 50:4417. doi: 10.1016/j.jacc.2007.03.052

95. Engel L-C, Landmesser U, Gigengack K, Wurster T, Manes C, Girke G, et al. Novel Approach for in vivo detection of vulnerable coronary plaques using molecular 3-T CMR imaging with an albumin-binding probe. JACC Cardiovasc Imaging. (2018) 12:297-306. doi: 10.1016/j.jcmg.2017.10.026

96. Xie Y, Pang J, Kim Y, Yang Q, Kim J-S, Nguyen CT, et al. Coronary atherosclerosis T1-weighed Characterization with integrated anatomical reference (CATCH). J Cardiovasc Magn Reson. (2016) 18:O22. doi: 10.1186/1532-429X-18-S1-O22
97. Xie Y, Kim YJ, Pang J, Kim JS, Yang Q, Wei J, et al. Coronary atherosclerosis T1-weighed characterization with integrated anatomical reference: comparison with high-risk plaque features detected by invasive coronary imaging. JACC Cardiovasc Imaging. (2017) 10:637-48. doi: 10.1016/j.jcmg.2016.06.014

98. Ginami G, Neji R, Phinikaridou A, Whitaker J, Botnar RM, Prieto C. Simultaneous bright- and black-blood whole-heart MRI for noncontrast enhanced coronary lumen and thrombus visualization. Magn Reson Med. (2018) 79:1460-72. doi: 10.1002/mrm.26815

99. Hartley A, Haskard D, Khamis R. Oxidized LDL and anti-oxidized LDL antibodies in atherosclerosis - novel insights and future directions in diagnosis and therapy Trends Cardiovasc Med. (2019) 29:22-6. doi: 10.1016/j.tcm.2018.05.010

100. Pandey SS, Haskard DO, Khamis RY. Developing a strategy for interventional molecular imaging of oxidized lowdensity lipoprotein in atherosclerosis. Mol Imaging. (2017) 16:1536012117723788. doi: 10.1177/1536012117723788

101. Khamis RY, Woollard KJ, Hyde GD, Boyle JJ, Bicknell C, Chang S-H, et al. Near Infrared Fluorescence (NIRF) molecular imaging of oxidized LDL with an autoantibody in experimental atherosclerosis. Sci Rep. (2016) 6:21785. doi: 10.1038/srep21785

102. Bary C Von, Makowski M, Preissel A, Keithahn V, Warley A, Spuentrup E, et al. MRI of coronary wall remodeling in a swine model of coronary injury using an elastin-binding contrast agent. Circ Cardiovasc Imaging. (2011) 4:147-5. doi: 10.1161/CIRCIMAGING.109. 895607

103. Caravan P, Das B, Dumas S, Epstein FH, Helm PA, Jacques V, et al. Collagen-targeted MRI contrast agent for molecular imaging of fibrosis. Angew Chemie Int Ed. (2007) 46:8171-73. doi: 10.1002/anie.2007 00700

104. Sanders HMHF, Strijkers GJ, Mulder WJM, Huinink HP, Erich SJF, Adan OCG, et al. Morphology, binding behavior and MR-properties of paramagnetic collagen-binding liposomes. Contrast Media Mol Imaging. (2009) 4:81-8. doi: 10.1002/cmmi.266

105. Hellenthal FAMVI, Buurman WA, Wodzig WKWH, Schurink GWH. Biomarkers of AAA progression. Part 1: extracellular matrix degeneration. Nat Rev Cardiol. (2009) 6:464-74. doi: 10.1038/nrcardio. 2009.80

106. Bazeli R, Coutard M, Duport BD, Lancelot E, Corot C, Laissy J$\mathrm{P}$, et al. In vivo evaluation of a new magnetic resonance imaging contrast agent (P947) to target matrix metalloproteinases in expanding experimental abdominal aortic aneurysms. Invest Radiol. (2010) 45:6628. doi: 10.1097/RLI.0b013e3181ee5bbf

Conflict of Interest: The authors declare that the research was conducted in the absence of any commercial or financial relationships that could be construed as a potential conflict of interest.

Publisher's Note: All claims expressed in this article are solely those of the authors and do not necessarily represent those of their affiliated organizations, or those of the publisher, the editors and the reviewers. Any product that may be evaluated in this article, or claim that may be made by its manufacturer, is not guaranteed or endorsed by the publisher.

Copyright (c) 2021 Hajhosseiny, Munoz, Cruz, Khamis, Kim, Prieto and Botnar. This is an open-access article distributed under the terms of the Creative Commons Attribution License (CC BY). The use, distribution or reproduction in other forums is permitted, provided the original author(s) and the copyright owner(s) are credited and that the original publication in this journal is cited, in accordance with accepted academic practice. No use, distribution or reproduction is permitted which does not comply with these terms. 